\title{
The determination of polycyclic aromatic hydrocarbons in some foods from industrialized areas in South Eastern Nigeria: human health risk impact
}

\author{
Uchechi Bliss ONYEDIKACHI*, Chuka Donatus BELONWU, and Mattew Owhonda WEGWU \\ Environmental Toxicology Unit, Department of Biochemistry, Faculty of Science, University of Port Harcourt, River \\ State, Nigeria
}

\begin{abstract}
This study investigated the potential health risk due to dietary exposure to Polycyclic Aromatic Hydrocarbons (PAHs) for the populace living around selected industrial areas in the south eastern states of Nigeria. The concentrations of PAHs were measured in edible food crops using Gas Chromatography - Flame Ionization Detection (GC-FID). The mean concentration of PAHs in food crops collected ranged from < 0.01 to $2.64 \pm 0.02,5.27 \pm 0.04,0.96 \pm 0.02,8.94 \pm$ 0.01 and $1.95 \pm 0.06 \mathrm{in} \mathrm{mg/kg}$ for Osisioma, Ishiagu, Irete, Akwuuru, and Ngwo respectively. PAHs distributions in food samples analyzed in this study showed vegetables to be significant higher $(p \leq 0.05)$ in the order vegetables $>$ fruits > nuts > tubers. Total PAHs ( $\sum \mathrm{PAHs}$ ) concentrations in most crop samples had highest values 14.49, 36.29, 4.59, 23.36 and $21.8 \mathrm{mg} / \mathrm{kg}$ for Chyrysophyllum albidum, Telferia occidentalis, Vernonia amygdalina, Talinum triangulare and Elaies guinnensis for Osisioma, Akwuuru, Irete, Ishiagu and Ngwo respectively. The low molecular weight-PAHS/ high molecular weight-PAHS were $<1$ with values $0.76,0.18,0.28,0.91$ and 0.12 for Osisioma, Irete, Akwuuru, Ishiagu and Ngwo respectively. $58.3 \%$ and $71.7 \%$ of $\sum \mathrm{B}(\mathrm{a}) \mathrm{P}$ and $\sum \mathrm{PAHs}$ in food samples in the study areas exceeded the permissible limits set by DPR and EU. The estimated daily intake of PAHs via the ingestion of food crops were within the tolerable range. The carcinogenic risk values for the food crops were within the predicted permissible lifetime risks of carcinogen $\left(10^{-6}-10^{-4}\right)$ for adults and children in Osisioma, Akwuuru, Ishiagu and Enugu respectively. Therefore, this study suggests that the populace around industrialized areas in South Eastern Nigeria, may be at risk due to PAHs exposure through food consumption.
\end{abstract}

Keywords: polycyclic aromatic hydrocarbons (PAHs); incremental life time cancer risk (ILCR); benzo(a)pyrene toxicity equivalence (TEBaP).

\section{Introduction}

Food safety is increasingly becoming a global challenge, and this has alerted several researches regarding the dangers incurred through ingestion of foods contaminated by toxins $[1,2]$. The toxicity of polycyclic aromatic hydrocarbons (PAHs) has gained serious interest among international regulatory bodies based on the degree of occurrence and toxicity [3], environmental biochemists, chemists, ecotoxicologists and government agencies [4].

PAHs refer to a class of heterogeneous organic compounds made up of two or more aromatic fused rings composed mainly of hydrocarbon with the absence of hetero-atoms or substituent in their structures [4, 5]. PAHs are generally lipophilic, volatile and not soluble in water but able organic matters [6]. Their solubility in water increases as molecular mass decreases in a linear fashion $[4,7]$. PAHs are pollutants formed when organic matters are burnt in the limited supply of oxygen. Examples of organic materials are coal, oil, gas, wood, garbage, tobacco, petroleum products etc. They are released into the environment mainly through anthropogenic activities (engine exhausts, industrial production processes like mining, petroleum refining/ petroleum distillation, coal-derived products, waste incineration, tobacco smoking and partly by natural means, e.g. bush burning and volcanic eruptions [4]. An increase in environmental PAHs levels is the major cause of high PAHs levels in food within industrialized and developing countries like Nigeria. Human exposure to PAHs can be through diet as PAHs are possible contaminants in fruits, vegetables, oils and dairy products. As a result, humans are exposed to several health problems which could be immunological, hematological, cardiological and hepatological [3]. PAHs dispersed into environment, i.e. air, water, sediments, soil and plants, result in the pollution of organisms in different habitats. Thus, possibly exposing humans to high PAHs levels through food supply especially in nonsmokers [6].

Some other studies show that PAHs play significant roles in the physiology of experimental animals as carcinogenes, mutagenes, and genotoxins [6]. Increased human risk to various forms of cancer (lungs, skin and bladder) is associated with dietary exposure to high levels of PAHs [6, 8]. Therefore, evaluating the cancer risk level of a given area via the ingestion of PAHs is important for effective environmental management and human risk assessment. Based on the levels of occurrence

*Corresponding author. E-mail address: ucheonyedi@gmail.com; ub.onyedikachi@mouau.edu.ng (Uchechi Bliss Onyedikachi). 
and carcinogenicity, the United States Environmental Protection Agency (USEPA) has highlighted sixteen. PAHs according to their precedence as: benzo(a)pyrene (BaP), benzo(a)anthracene (BaA), dibenzo(b)fluoranthene (BbF), chrysene (Chry), benzo(k)fluoranthene $(\mathrm{BkF})$, indeno(1,2,3-cd)perylene (IndP) and dibenzo(a,h)anthracene (DahA), considered as human carcinogens, and others like of naphthalene (Nap), acenaphthene (Ace), acenaphthylene (Acy), anthracene (Ant), benzo(ghi)perylene (BghiP), fluoranthene (Flt), fluorene (Flu), phenanthrene (Phe) and pyrene (Pyr), noted as non-carcinogenic PAHs [3, 6]. The toxicity of PAHs in food as well as the carcinogenic potential of these compounds to humans via intake explains why it is of utmost importance to monitor their presence in food [6].

Availability of safe and healthy food to residents living around the industrial zones in the south east of Nigeria has become a serious environmental problem. There is no cogent information as regards PAHs contamination level and its health risk implication to the populace of these study areas. The increase in contamination of soils and crops by PAHs around these zones through industrial activities may lead to increase in the consumption of PAHs. Thus, the main aim of this present study were:

(1) To investigate the concentrations of 16 priority PAHs in selected vegetables, tubers, fruits and nuts collected from south eastern states of Nigeria (Osisioma, Akwuuru, Ngwo, Ishiagu and Irete);

(2) To evaluate the health risk associated with the dietary intake PAHs through the ingestion of food crops;

(3) To assess the levels and sources of PAHs pollution using diagnostic ratios.

\section{Experimental}

\subsection{Materials and methods}

Five samples each of twelve different food crops which includes: (i) vegetables, i.e. bitter leaf (Vernonia amygdalina), water leaf (Talinum triangulare) and pumpkin leaf (Telfairia occidentalis); (ii) tubers, i.e. yam (Dioscorea alata), cocoyam (Colocasia esculenta) and cassava (Manihot esculenta); (iii) fruits, included orange (Citrus sinensis), paw paw (Carica papaya), star apple (Chrysophyllum albidum); (iv) nuts, i.e. kola nut (Cola acumulata), palm kernel nut (Elaeis guineensis jacq) and coconut (Cocos nucifera), were harvested from farmlands close to the industries (study sites) at Osisioma, Akwuuru, Ishiagu, Ngwo, and Irete. Umudike (a university farmland devoid of industries) was the control for this study.

The crop samples were washed with water three times to avoid contamination. After the water had evaporated, the vegetables were plucked, selected and spread-out on a flat foiled surface. The skin of the tubers peeled and chopped in to tiny cubes for easy drying, the fruits were peeled to remove exocarp (skin) while endocarp (flesh) was collected. The flesh of the nuts was collected and chopped into tiny cubes (the hard shells of coconut and palm kernel nut were removed to access the flesh although this was not needed for the kola nut). Each sample was weighed, oven dried at $55{ }^{\circ} \mathrm{C}$ for $48 \mathrm{~h}$ and crushed into powder.

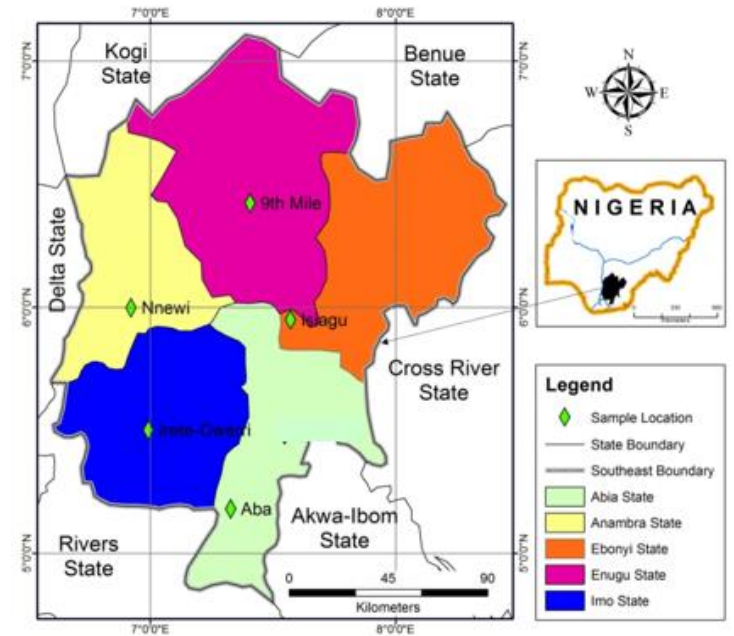

Figure 1. Map of the south eastern states of Nigeria showing some industrial areas of study.

Extraction. $2 \mathrm{~g}$ of each plant sample were weighed into a clean extraction container. After which, $10 \mathrm{ml}$ of dichloromethane was added to it and stirred vigorously and then allowed to settle. The mixture was filtered and concentrated to $2 \mathrm{ml}$ [9].

Sample cleanup/separation. $1 \mathrm{~cm}$ of moderately packed glass wool was placed at the bottom of $10 \mathrm{~mm} \mathrm{ID} * 250$ $\mathrm{mm}$ Loup chromatographic column. Slurry of $2 \mathrm{~g}$ activated silica was added to $10 \mathrm{ml}$ methylene chloride and was placed into the chromatographic column. $0.5 \mathrm{~cm}$ of sodium sulfate was added to the column. The column was rinsed with additional $10 \mathrm{ml}$ methylene chloride and pre-eluted with $20 \mathrm{ml}$ of dichloromethane. This was allowed to flow through the column at a rate of about 2 min until the liquid in the column was just above the sulfate layer.

The extraction bottle was rinsed with $1 \mathrm{ml}$ of dichloromethane and added to the column as well. The stop clock of the column was opened, and the element was collected. Following the recovery of the labeled fractions called "Aliphatics" before the exposure of the sodium sulfate layer, the column was eluted with $1: 1$ mixture of propanone and dichlorometane in $1-2 \mathrm{ml}$ increments. Another $8-10 \mathrm{ml}$ of eluent were collected [9 - 12].

Gas chromatography analysis. Gas chromatography flame ionization detector (GC-FID) (Hewlett Packard, Wilmington, DE, USA), powered with HP chemstation Rev. A 09:01 (10206) software was used to identify and quantify compounds. The GC operating conditions were as follow: fused silica column [30m*0.25 $\mu \mathrm{m}$ film of HP5 (thickness)]; the inlet and injection temperature was set at $270{ }^{\circ} \mathrm{C}$ to $320{ }^{\circ} \mathrm{C}$. Split injection was adopted with a split ratio of 8:1 using a rubber septum and $1 \mu \mathrm{l}$ of the concentrated sample was injected into the column.

The column temperature was programmed as follow: hold at $65^{\circ} \mathrm{C}$ for $2 \mathrm{~min} ; 65-260^{\circ} \mathrm{C}$ at $12{ }^{\circ} \mathrm{C} / \mathrm{min} ; 260-$ $320^{\circ} \mathrm{C}$ at $15^{\circ} \mathrm{C} / \mathrm{min}$ and maintained at $310^{\circ} \mathrm{C}$ for $8 \mathrm{~min}$. The oven temperature was set at $65{ }^{\circ} \mathrm{C}$. Nitrogen was used as carrier gas $(30 \mathrm{ml} / \mathrm{min})$. The hydrogen and compressed air pressure was 27.8 psi at $35 \mathrm{ml} / \mathrm{min}$ and 
$250 \mathrm{ml} / \mathrm{min}$ respectively. A standard mixture of the 16 PAHs was used for the PAHs analysis. Comparison between the retention time of standards and that of the extracts was obtained to identify the compounds while individual PAHs analysis were used for the quantification. To ensure accuracy for all the PAHs measured, the method of Tango et al. [13] was used. The recovery of internal standard was used to ascertain the analytical method used for the PAHs.

\subsection{Quality control}

Quality control was observed at all times during the entire course of this research. Analyses of procedural blanks were periodically carried out to ascertain quality of analytical results devoid of laboratory contamination and operational errors. The average blank concentration was subtracted from each sample to correct for methodological and equipment errors. The detection limit and quantitation limits were calculated as 3 and 10 times the standard deviation of the blank, respectively. The recoveries of each individual $\mathrm{PAH}$ varied from 93.14\% to $104.92 \%$ for PHE and DahA, respectively. The calibration curves were obtained through serial dilution of stock standard solutions with $n$-hexane to obtain 6 calibration standard PAHs solutions containing $5,10,20,30,40$, and $50 \mu \mathrm{g} / \mathrm{L}$ of stock solution. The calibration curves for PAHs analyzed standards $(n=6)$ gave values of remaining standard deviations that ranged between $78.05 \%$ and $101.20 \%$, demonstrating analytical method based on its repeatability. Triplicate determinations were made on all food crops samples [11, $13]$.
Statistical analysis. The one-way ANOVA, using computer aided SPSS package (version 20.0) was used to statistically analyze the data gotten in this study. Test of statistical significance was done at $95 \%$ confidence level. Data was reported the mean concentration of PAHs \pm standard error (S.E.) of mean.

\subsection{Health risk dietary exposure estimates}

The toxic potency assessment of PAHs is estimated as the concentration of each PAH congener in a sample multiplied by its Benzo[a]P toxic equivalent factor according to method of Tongo et al. [11] using the equation given below:

$$
B[a] \text { Pteq }=C i \times T E F i
$$

The Toxic Equivalence Quotient (TEQ) is the toxic potency of PAHs in the sample environment obtained by summing the toxic potencies of individual PAHs (B[a]Pteq) using equation given below:

$$
T E Q=\Sigma B[a] \text { Pteq }
$$

Concentrations of Benzo(a)pyrene equivalents (B[a]Peq) in food crops and the daily intake (DI) of PAHs via consumption of food crops were estimated using Equations 2 and 3

$$
D I=\sum_{i=1}^{n} B C i x F i
$$

where: $\mathrm{B}$ is the concentration of $\mathrm{B}[\mathrm{a}] \mathrm{Peq}$ in food crops $(\mathrm{mg} / \mathrm{kg}) ; \mathrm{C} i$ is the concentration of PAHS congener $i$ in food sample; TEF $i=$ toxic equivalent factors (TEFs) of PAHS congener $i ; F i$ is the food intake per-day (g/d) [14].

\section{Results and discussion}

\begin{tabular}{|c|c|c|c|c|c|c|c|c|c|c|c|c|c|}
\hline & & VA & TO & TT & ME & DR & CA & CS & CHA & $\mathbf{C P}$ & $\mathrm{CN}$ & $\mathrm{CO}$ & EG \\
\hline \multirow[t]{5}{*}{ NAP } & Abia & $0.06 \pm 0.01 \mathrm{a}$ & $0.68 \pm 0.02 b$ & $0.16 \pm 0.00 \mathrm{c}$ & $0.05 \pm 0.00 \mathrm{a}$ & $0.00 \pm 0.00 \mathrm{~d}$ & $0.00 \pm 0.00 \mathrm{~d}$ & $1.09 \pm 0.02 \mathrm{e}$ & $2.64 \pm 0.02 \mathrm{f}$ & $0.00 \pm 0.00 \mathrm{~d}$ & $0.00 \pm 0.00 \mathrm{~d}$ & $0.00 \pm 0.00 \mathrm{~d}$ & $\overline{0.07 \pm 0.00 a}$ \\
\hline & Owerri & $0.06 \pm 0.01 \mathrm{a}$ & $0.00 \pm 0.00 \mathrm{~b}$ & $0.00 \pm 0.00 \mathrm{~b}$ & $0.00 \pm 0.00 \mathrm{~b}$ & $0.00 \pm 0.00 \mathrm{~b}$ & $0.00 \pm 0.00 \mathrm{~b}$ & $0.07 \pm 0.00 \mathrm{a}$ & $0.05 \pm 0.01 \mathrm{a}$ & $0.04 \pm 0.01 \mathrm{a}$ & $0.07 \pm 0.01 \mathrm{a}$ & $0.00 \pm 0.00 \mathrm{~b}$ & $0.03 \pm 0.01 \mathrm{c}$ \\
\hline & Anambra & $0.28 \pm 0.02 \mathrm{a}$ & $0.35 \pm 0.01 \mathrm{a}$ & $0.73 \pm 0.07 \mathrm{~b}$ & $0.11 \pm 0.01 \mathrm{c}$ & $0.19 \pm 0.01 \mathrm{c}$ & $0.00 \pm 0.00 \mathrm{~d}$ & $0.00 \pm 0.00 \mathrm{~d}$ & $0.02 \pm 0.00 \mathrm{~d}$ & $0.00 \pm 0.00 \mathrm{~d}$ & $0.00 \pm 0.00 \mathrm{~d}$ & $0.20 \pm 0.01 \mathrm{a}$ & $0.00 \pm 0.00 \mathrm{~d}$ \\
\hline & Ebonyi & $0.11 \pm 0.01 \mathrm{a}$ & $0.99 \pm 0.01 \mathrm{~b}$ & $0.70 \pm 0.00 \mathrm{c}$ & $0.00 \pm 0.00 \mathrm{~d}$ & $0.00 \pm 0.00 \mathrm{~d}$ & $0.01 \pm 0.00 \mathrm{~d}$ & $0.00 \pm 0.00 \mathrm{~d}$ & $0.16 \pm 0.01 \mathrm{a}$ & $0.03 \pm 0.00 \mathrm{~d}$ & $0.15 \pm 0.01 \mathrm{a}$ & $0.01 \pm 0.00 \mathrm{~d}$ & $0.04 \pm 0.00 \mathrm{~d}$ \\
\hline & Enugu & $0.06 \pm 0.01 \mathrm{a}$ & $0.01 \pm 0.00^{\mathrm{b}}$ & $0.00 \pm 0.00 \mathrm{c}$ & $0.06 \pm 0.00 \mathrm{a}$ & $0.16 \pm 0.00 \underline{\underline{d}}$ & $0.06 \pm 0.00 \mathrm{a}$ & $0.00 \pm 0.00 \mathrm{c}$ & $0.00 \pm 0.00 \mathrm{c}$ & $0.00 \pm 0.00 \mathrm{c}$ & $0.00 \pm 0.00 \mathrm{c}$ & $0.00 \pm 0.00 \mathrm{c}$ & $0.10 \pm 0.00 \mathrm{e}$ \\
\hline \multirow{5}{*}{$\begin{array}{l}\text { MET } \\
\text { NAP }\end{array}$} & Abia & $0.19 \pm 0.01 \mathrm{a}$ & $3.54 \pm 0.02 \mathrm{~b}$ & $0.23 \pm 0.00 \mathrm{a}$ & $0.02 \pm 0.00 \mathrm{c}$ & $0.00 \pm 0.00 \mathrm{c}$ & $0.00 \pm 0.00 \mathrm{c}$ & $0.28 \pm 0.02 \mathrm{a}$ & $0.89 \pm 0.02 \mathrm{~d}$ & $0.00 \pm 0.00 \mathrm{c}$ & $0.00 \pm 0.00 \mathrm{c}$ & $0.00 \pm 0.00 \mathrm{c}$ & $0.05 \pm 0.01 \mathrm{c}$ \\
\hline & Owerri & $0.06 \pm 0.01 \mathrm{a}$ & $0.09 \pm 0.01 \mathrm{a}$ & $0.00 \pm 0.00 \mathrm{~b}$ & $0.00 \pm 0.00 \mathrm{~b}$ & $0.00 \pm 0.00 \mathrm{~b}$ & $0.00 \pm 0.00 \mathrm{~b}$ & $0.07 \pm 0.01 \mathrm{a}$ & $0.10 \pm 0.01 \mathrm{c}$ & $0.00 \pm 0.00 \mathrm{~b}$ & $0.04 \pm 0.01 \mathrm{a}$ & $0.00 \pm 0.00 \mathrm{~b}$ & $0.00 \pm 0.00 \mathrm{~b}$ \\
\hline & Anambra & $0.95 \pm 0.05 \mathrm{a}$ & $1.25 \pm 0.05 b$ & $1.00 \pm 0.00 \mathrm{~d}$ & $0.04 \pm 0.00 \mathrm{c}$ & $0.02 \pm 0.01 \mathrm{c}$ & $0.00 \pm 0.00 \mathrm{c}$ & $0.00 \pm 0.00 \mathrm{c}$ & $0.07 \pm 0.01 \mathrm{~d}$ & $0.00 \pm 0.00 \mathrm{c}$ & $0.00 \pm 0.00 \mathrm{c}$ & $0.09 \pm 0.01 \mathrm{a}$ & $0.00 \pm 0.00 \mathrm{c}$ \\
\hline & Ebonyi & $0.11 \pm 0.01 \mathrm{a}$ & $5.27 \pm 0.04 \mathrm{~b}$ & $1.05 \pm 0.01 \mathrm{c}$ & $0.00 \pm 0.00 \mathrm{~d}$ & $0.00 \pm 0.00 \mathrm{~d}$ & $0.02 \pm 0.00 \mathrm{~d}$ & $0.00 \pm 0.00 \mathrm{~d}$ & $0.06 \pm 0.01 \mathrm{a}$ & $0.00 \pm 0.00 \mathrm{~d}$ & $0.10 \pm 0.01 \mathrm{a}$ & $0.02 \pm 0.00 \mathrm{~d}$ & $0.02 \pm 0.01 \mathrm{~d}$ \\
\hline & Enugu & $0.20 \pm 0.01 \mathrm{a}$ & $0.00 \pm 0.00 \mathrm{~b}$ & $0.00 \pm 0.00 \mathrm{~b}$ & $0.03 \pm 0.00 \mathrm{c}$ & $0.02 \pm 0.01 \mathrm{c}$ & $0.12 \pm 0.01 \mathrm{~d}$ & $0.00 \pm 0.00 \mathrm{~b}$ & $0.00 \pm 0.00 \mathrm{~b}$ & $0.00 \pm 0.00 \mathrm{~b}$ & $0.00 \pm 0.00 \mathrm{~b}$ & $0.00 \pm 0.00 \mathrm{~b}$ & $0.02 \pm 0.01 \mathrm{c}$ \\
\hline \multirow[t]{5}{*}{$\mathrm{ACY}$} & Abia & $0.35 \pm 0.01 \mathrm{a}$ & $2.10 \pm 0.01 \mathrm{~b}$ & $0.26 \pm 0.00 \mathrm{a}$ & $0.07 \pm 0.00 \mathrm{c}$ & $0.00 \pm 0.00 \mathrm{~d}$ & $0.00 \pm 0.00 \mathrm{~d}$ & $0.22 \pm 0.01 \mathrm{a}$ & $1.00 \pm 0.01 \mathrm{~d}$ & $0.00 \pm 0.00 \mathrm{~d}$ & $0.00 \pm 0.00 \mathrm{~d}$ & $0.00 \pm 0.00 \mathrm{f}$ & $0.02 \pm 0.00 \mathrm{~d}$ \\
\hline & Owerri & $0.09 \pm 0.01 \mathrm{a}$ & $0.06 \pm 0.01 \mathrm{a}$ & $0.07 \pm 0.01 \mathrm{a}$ & $0.00 \pm 0.00 \mathrm{~b}$ & $0.00 \pm 0.00 \mathrm{~b}$ & $0.00 \pm 0.00 \mathrm{~b}$ & $0.05 \pm 0.01 \mathrm{a}$ & $0.02 \pm 0.00 \mathrm{a}$ & $0.03 \pm 0.01 \mathrm{a}$ & $0.02 \pm 0.01 \mathrm{a}$ & $0.00 \pm 0.00 \mathrm{~b}$ & $0.00 \pm 0.00 \mathrm{~b}$ \\
\hline & Anambra & $1.79 \pm 0.02 \mathrm{a}$ & $1.09 \pm 0.02 \mathrm{~b}$ & $1.15 \pm 0.05 b$ & $0.16 \pm 0.01 \mathrm{c}$ & $0.03 \pm 0.01 \mathrm{~d}$ & $0.06 \pm 0.00 \mathrm{~d}$ & $0.00 \pm 0.00 \mathrm{e}$ & $0.02 \pm 0.01 \mathrm{e}$ & $0.00 \pm 0.00 \mathrm{e}$ & $0.00 \pm 0.00 \mathrm{e}$ & $0.05 \pm 0.00$ & $0.07 \pm 0.01 \mathrm{~d}$ \\
\hline & Ebonyi & $0.17 \pm 0.01 \mathrm{a}$ & $3.12 \pm 0.01 \mathrm{~b}$ & $1.15 \pm 0.01 \mathrm{c}$ & $0.00 \pm 0.00 \mathrm{~d}$ & $0.00 \pm 0.00 \mathrm{~d}$ & $0.03 \pm 0.00 \mathrm{~d}$ & $0.00 \pm 0.00 \mathrm{~d}$ & $0.07 \pm 0.01 \mathrm{e}$ & $0.02 \pm 0.01 \mathrm{~d}$ & $0.04 \pm 0.01 \mathrm{~d}$ & $0.00 \pm 0.00 \mathrm{~d}$ & $0.02 \pm 0.01 \mathrm{~d}$ \\
\hline & Enugu & $0.39 \pm 0.01 \mathrm{a}$ & $0.00 \pm 0.00 \mathrm{~b}$ & $0.03 \pm 0.00 \mathrm{~b}$ & $0.09 \pm 0.01 \mathrm{c}$ & $0.02 \pm 0.00 \mathrm{~b}$ & $0.97 \pm 0.03 \mathrm{~d}$ & $0.00 \pm 0.00 \mathrm{~b}$ & $0.00 \pm 0.00 \mathrm{~b}$ & $0.01 \pm 0.00 \mathrm{~b}$ & $0.00 \pm 0.00 \mathrm{~b}$ & $0.00 \pm 0.00 \mathrm{~b}$ & $0.01 \pm 0.00 \mathrm{~b}$ \\
\hline \multirow[t]{5}{*}{ ACE } & Abia & $0.04 \pm 0.01 \mathrm{a}$ & $0.54 \pm 0.00 \mathrm{~b}$ & $0.18 \pm 0.01 \mathrm{c}$ & $0.08 \pm 0.00 \mathrm{a}$ & $0.00 \pm 0.00 \mathrm{~d}$ & $0.00 \pm 0.00 \mathrm{~d}$ & $0.12 \pm 0.00 \mathrm{c}$ & $0.25 \pm 0.01 \mathrm{~b}$ & $0.00 \pm 0.00 \mathrm{~d}$ & $0.00 \pm 0.00 \mathrm{~d}$ & $0.00 \pm 0.00 \mathrm{~d}$ & $0.00 \pm 0.00 \mathrm{~d}$ \\
\hline & Owerri & $0.09 \pm 0.01 \mathrm{a}$ & $0.01 \pm 0.00 \mathrm{~b}$ & $0.05 \pm 0.01 \mathrm{~b}$ & $0.00 \pm 0.00 \mathrm{~b}$ & $0.00 \pm 0.00 \mathrm{~b}$ & $0.00 \pm 0.00 \mathrm{~b}$ & $0.11 \pm 0.00 \mathrm{a}$ & $0.02 \pm 0.01 \mathrm{~b}$ & $0.01 \pm 0.00 \mathrm{~b}$ & $0.03 \pm 0.00 \mathrm{~b}$ & $0.00 \pm 0.00 \mathrm{~b}$ & $0.00 \pm 0.00 \mathrm{~b}$ \\
\hline & Anambra & $0.19 \pm 0.02 \mathrm{a}$ & $0.95 \pm 0.05 \mathrm{~b}$ & $0.87 \pm 0.14 \mathrm{~b}$ & $0.17 \pm 0.01 \mathrm{a}$ & $0.05 \pm 0.01 \mathrm{c}$ & $0.01 \pm 0.00 \mathrm{c}$ & $0.00 \pm 0.00 \mathrm{~d}$ & $0.00 \pm 0.00 \mathrm{~d}$ & $0.00 \pm 0.00 \mathrm{~d}$ & $0.00 \pm 0.00 \mathrm{~d}$ & $0.01 \pm 0.01 \mathrm{~d}$ & $0.01 \pm 0.00 \mathrm{~d}$ \\
\hline & Ebonyi & $0.17 \pm 0.01 \mathrm{a}$ & $0.90 \pm 0.10 \mathrm{~b}$ & $0.78 \pm 0.02 \mathrm{~b}$ & $0.00 \pm 0.00 \mathrm{c}$ & $0.00 \pm 0.00 \mathrm{c}$ & $0.06 \pm 0.01 \mathrm{~d}$ & $0.00 \pm 0.00 \mathrm{e}$ & $0.02 \pm 0.01 \mathrm{~d}$ & $0.01 \pm 0.00 \mathrm{e}$ & $0.08 \pm 0.01 \mathrm{~d}$ & $0.01 \pm 0.00 \mathrm{e}$ & $0.01 \pm 0.00 \mathrm{e}$ \\
\hline & Enugu & $0.05 \pm 0.01 \mathrm{a}$ & $0.00 \pm 0.00 \mathrm{~b}$ & $0.04 \pm 0.02 \mathrm{a}$ & $0.10 \pm 0.00 \mathrm{c}$ & $0.04 \pm 0.01 \mathrm{a}$ & $0.48 \pm 0.03 \mathrm{~d}$ & $0.00 \pm 0.00 \mathrm{~b}$ & $0.00 \pm 0.00 \mathrm{~b}$ & $0.02 \pm 0.01 \mathrm{~b}$ & $0.00 \pm 0.00 \mathrm{~b}$ & $0.00 \pm 0.00 \mathrm{~b}$ & $0.02 \pm 0.00 \mathrm{~b}$ \\
\hline \multirow[t]{5}{*}{ FLO } & Abia & $0.19 \pm 0.01 \mathrm{a}$ & $0.97 \pm 0.04 \mathrm{~b}$ & $0.39 \pm 0.02 \mathrm{c}$ & $0.13 \pm 0.00 \mathrm{a}$ & $0.00 \pm 0.00 \mathrm{~d}$ & $0.00 \pm 0.00 \mathrm{~d}$ & $1.33 \pm 0.02 \mathrm{e}$ & $0.87 \pm 0.03 \mathrm{~b}$ & $0.00 \pm 0.00 \mathrm{~d}$ & $0.00 \pm 0.00 \mathrm{~d}$ & $0.00 \pm 0.00 \mathrm{~d}$ & $0.02 \pm 0.01 \mathrm{~d}$ \\
\hline & Owerri & $0.02 \pm 0.01 \mathrm{a}$ & $0.09 \pm 0.02 \mathrm{~b}$ & $0.09 \pm 0.01 \mathrm{~b}$ & $0.00 \pm 0.00 \mathrm{c}$ & $0.00 \pm 0.00 \mathrm{c}$ & $0.00 \pm 0.00 \mathrm{c}$ & $0.13 \pm 0.01 \mathrm{~d}$ & $0.14 \pm 0.01 \mathrm{~d}$ & $0.01 \pm 0.00 \mathrm{a}$ & $0.05 \pm 0.01 \mathrm{~b}$ & $0.00 \pm 0.00 \mathrm{c}$ & $0.04 \pm 0.00 \mathrm{~b}$ \\
\hline & Anambra & $0.94 \pm 0.01 \mathrm{a}$ & $0.99 \pm 0.02 \mathrm{a}$ & $1.70 \pm 0.10 \mathrm{~b}$ & $0.28 \pm 0.01 \mathrm{c}$ & $0.04 \pm 0.00 \mathrm{~d}$ & $0.10 \pm 0.01 \mathrm{e}$ & $0.00 \pm 0.00 \mathrm{~d}$ & $0.09 \pm 0.01 \mathrm{e}$ & $0.00 \pm 0.00 \mathrm{~d}$ & $0.00 \pm 0.00 \mathrm{~d}$ & $0.03 \pm 0.00 \mathrm{~d}$ & $0.05 \pm 0.01 \mathrm{~d}$ \\
\hline & Ebonyi & $0.03 \pm 0.01 \mathrm{a}$ & $1.39 \pm 0.02 \mathrm{~b}$ & $1.74 \pm 0.07 \mathrm{~b}$ & $0.00 \pm 0.00 \mathrm{c}$ & $0.00 \pm 0.00 \mathrm{c}$ & $0.08 \pm 0.01 \mathrm{~d}$ & $0.00 \pm 0.00 \mathrm{c}$ & $0.06 \pm 0.01 \mathrm{~d}$ & $0.01 \pm 0.00 \mathrm{a}$ & $0.09 \pm 0.01 \mathrm{~d}$ & $0.01 \pm 0.00 \mathrm{a}$ & $0.09 \pm 0.01 \mathrm{~d}$ \\
\hline & Enugu & $0.21 \pm 0.01 \mathrm{a}$ & $0.00 \pm 0.00 \mathrm{~b}$ & $0.06 \pm 0.01 \mathrm{c}$ & $0.17 \pm 0.01 \mathrm{~d}$ & $0.05 \pm 0.01 \mathrm{c}$ & $0.30 \pm 0.01 \mathrm{a}$ & $0.00 \pm 0.00 \mathrm{~b}$ & $0.00 \pm 0.00 \mathrm{~b}$ & $0.06 \pm 0.01 \mathrm{c}$ & $0.00 \pm 0.00 \mathrm{~b}$ & $0.00 \pm 0.00 \mathrm{~b}$ & $0.20 \pm 0.01 \mathrm{a}$ \\
\hline \multirow[t]{5}{*}{ PHE } & Abia & $0.39 \pm 0.01 \mathrm{a}$ & $0.69 \pm 0.02 \mathrm{~b}$ & $0.33 \pm 0.02 \mathrm{a}$ & $0.15 \pm 0.01 \mathrm{c}$ & $0.00 \pm 0.00 \mathrm{~d}$ & $0.00 \pm 0.00 \mathrm{~d}$ & $0.78 \pm 0.03 b$ & $0.63 \pm 0.02 b$ & $0.00 \pm 0.00 \mathrm{~d}$ & $0.00 \pm 0.00 \mathrm{~d}$ & $0.00 \pm 0.00 \mathrm{~d}$ & $0.02 \pm 0.01 \mathrm{~d}$ \\
\hline & Owerri & $0.14 \pm 0.01 \mathrm{a}$ & $0.56 \pm 0.00 \mathrm{~b}$ & $0.00 \pm 0.00 \mathrm{c}$ & $0.00 \pm 0.00 \mathrm{c}$ & $0.00 \pm 0.00 \mathrm{c}$ & $0.00 \pm 0.00 \mathrm{c}$ & $0.04 \pm 0.02 \mathrm{c}$ & $0.12 \pm 0.00 \mathrm{a}$ & $0.03 \pm 0.01 \mathrm{c}$ & $0.02 \pm 0.00 \mathrm{c}$ & $0.00 \pm 0.00 \mathrm{c}$ & $0.08 \pm 0.01 \mathrm{a}$ \\
\hline & Anambra & $1.97 \pm 0.02 \mathrm{a}$ & $0.28 \pm 0.03 b$ & $1.42 \pm 0.08 \mathrm{a}$ & $0.31 \pm 0.01 \mathrm{~b}$ & $0.02 \pm 0.00 \mathrm{c}$ & $0.14 \pm 0.02 \mathrm{~d}$ & $0.00 \pm 0.00 \mathrm{e}$ & $0.09 \pm 0.01 \mathrm{~d}$ & $0.00 \pm 0.00 \mathrm{c}$ & $0.00 \pm 0.00 \mathrm{c}$ & $0.00 \pm 0.00 \mathrm{c}$ & $0.30 \pm 0.01 \mathrm{~b}$ \\
\hline & Ebonyi & $0.25 \pm 0.00 \mathrm{a}$ & $1.01 \pm 0.01 \mathrm{~b}$ & $1.45 \pm 0.05 \mathrm{c}$ & $0.00 \pm 0.00 \mathrm{~d}$ & $0.00 \pm 0.00 \mathrm{~d}$ & $0.05 \pm 0.01 \mathrm{~d}$ & $0.00 \pm 0.00 \mathrm{~d}$ & $0.05 \pm 0.01 \mathrm{~d}$ & $0.01 \pm 0.00 \mathrm{~d}$ & $0.06 \pm 0.01 \mathrm{~d}$ & $0.02 \pm 0.01 \mathrm{~d}$ & $0.17 \pm 0.01 \mathrm{e}$ \\
\hline & Enugu & $0.42 \pm 0.01 \mathrm{a}$ & $0.03 \pm 0.00 \mathrm{~b}$ & $0.00 \pm 0.00 \mathrm{c}$ & $0.18 \pm 0.01 \mathrm{~d}$ & $0.02 \pm 0.00 \mathrm{~b}$ & $0.08 \pm 0.01 \mathrm{c}$ & $0.00 \pm 0.00 \mathrm{c}$ & $0.00 \pm 0.00$ & $0.03 \pm 0.00 \mathrm{c}$ & $0.00 \pm 0.00 \mathrm{c}$ & $0.00 \pm 0.00 \mathrm{c}$ & $0.39 \pm 0.02 \mathrm{a}$ \\
\hline \multirow[t]{5}{*}{ ANT } & Abia & $0.42 \pm 0.01 \mathrm{a}$ & $1.42 \pm 0.08 \mathrm{~b}$ & $0.59 \pm 0.01 \mathrm{a}$ & $0.10 \pm 0.01 \mathrm{c}$ & $0.00 \pm 0.00 \mathrm{~d}$ & $0.00 \pm 0.00 \mathrm{~d}$ & $0.55 \pm 0.01 \mathrm{a}$ & $0.48 \pm 0.02 \mathrm{a}$ & $0.00 \pm 0.00 \mathrm{~d}$ & $0.00 \pm 0.00 \mathrm{~d}$ & $0.00 \pm 0.00 \mathrm{~d}$ & $0.02 \pm 0.01 \mathrm{~d}$ \\
\hline & Owerri & $0.41 \pm 0.01 \mathrm{a}$ & $0.51 \pm 0.01 \mathrm{a}$ & $0.08 \pm 0.01 \mathrm{~b}$ & $0.00 \pm 0.00 \mathrm{c}$ & $0.00 \pm 0.00 \mathrm{c}$ & $0.00 \pm 0.00 \mathrm{c}$ & $0.07 \pm 0.02 \mathrm{~b}$ & $0.10 \pm 0.01 \mathrm{~b}$ & $0.03 \pm 0.00 \mathrm{c}$ & $0.02 \pm 0.00 \mathrm{c}$ & $0.00 \pm 0.00 \mathrm{c}$ & $0.07 \pm 0.01 \mathrm{~b}$ \\
\hline & Anambra & $2.15 \pm 0.01 \mathrm{a}$ & $1.88 \pm 0.03 \mathrm{~b}$ & $2.49 \pm 0.01 \mathrm{a}$ & $0.21 \pm 0.00 \mathrm{c}$ & $0.03 \pm 0.01 \mathrm{~d}$ & $0.14 \pm 0.02 \mathrm{e}$ & $0.00 \pm 0.00 \mathrm{~d}$ & $0.06 \pm 0.01 \mathrm{~d}$ & $0.00 \pm 0.00 \mathrm{~d}$ & $0.00 \pm 0.00 \mathrm{~d}$ & $0.01 \pm 0.01 \mathrm{~d}$ & $0.17 \pm 0.01 \mathrm{e}$ \\
\hline & Ebonyi & $0.80 \pm 0.01 \mathrm{a}$ & $2.00 \pm 0.01 \mathrm{~b}$ & $2.59 \pm 0.01 \mathrm{~b}$ & $0.00 \pm 0.00 \mathrm{c}$ & $0.00 \pm 0.00 \mathrm{c}$ & $0.08 \pm 0.01 \mathrm{c}$ & $0.00 \pm 0.00 \mathrm{c}$ & $0.04 \pm 0.01 \mathrm{c}$ & $0.03 \pm 0.01 \mathrm{c}$ & $0.05 \pm 0.01 \mathrm{c}$ & $0.01 \pm 0.00 \mathrm{c}$ & $0.14 \pm 0.02 \mathrm{~d}$ \\
\hline & Enugu & $0.48 \pm 0.03 \mathrm{a}$ & $0.06 \pm 0.01 \mathrm{~b}$ & $0.04 \pm 0.00 \mathrm{~b}$ & $0.13 \pm 0.02 \mathrm{c}$ & $0.01 \pm 0.00 \mathrm{~b}$ & $0.25 \pm 0.01 \mathrm{~d}$ & $0.00 \pm 0.00 \mathrm{~b}$ & $0.00 \pm 0.00 \mathrm{~b}$ & $0.04 \pm 0.01 \mathrm{~b}$ & $0.00 \pm 0.00 \mathrm{~b}$ & $0.00 \pm 0.00 \mathrm{~b}$ & $0.29 \pm 0.01 \mathrm{~d}$ \\
\hline \multirow[t]{5}{*}{ FLA } & Abia & $0.25 \pm 0.01 \mathrm{a}$ & $1.57 \pm 0.04 \mathrm{~b}$ & $0.39 \pm 0.01 \mathrm{a}$ & $0.09 \pm 0.01 \mathrm{c}$ & $0.00 \pm 0.00 \mathrm{e}$ & $0.00 \pm 0.00 \mathrm{e}$ & $1.33 \pm 0.02 \mathrm{~b}$ & $2.84 \pm 0.02 \mathrm{~d}$ & $0.00 \pm 0.00 \mathrm{e}$ & $0.00 \pm 0.00 \mathrm{e}$ & $0.00 \pm 0.00 \mathrm{e}$ & $0.34 \pm 0.02 \mathrm{a}$ \\
\hline & Owerri & $0.96 \pm 0.02 \mathrm{a}$ & $1.32 \pm 0.01 \mathrm{~b}$ & $0.02 \pm 0.00 \mathrm{c}$ & $0.00 \pm 0.00 \mathrm{c}$ & $0.00 \pm 0.00 \mathrm{c}$ & $0.00 \pm 0.00 \mathrm{c}$ & $0.34 \pm 0.01 \mathrm{~d}$ & $0.22 \pm 0.00 \mathrm{~d}$ & $0.20 \pm 0.00 \mathrm{~d}$ & $0.02 \pm 0.01 \mathrm{c}$ & $0.00 \pm 0.00 \mathrm{c}$ & $2.40 \pm 0.10 \mathrm{e}$ \\
\hline & Anambra & $1.25 \pm 0.01 \mathrm{a}$ & $0.68 \pm 0.02 \mathrm{~b}$ & $1.63 \pm 0.02 \mathrm{a}$ & $0.19 \pm 0.01 \mathrm{~b}$ & $0.09 \pm 0.01 \mathrm{c}$ & $0.13 \pm 0.00 \mathrm{~b}$ & $0.00 \pm 0.00 \mathrm{~d}$ & $0.15 \pm 0.01 \mathrm{~b}$ & $0.00 \pm 0.00 \mathrm{~d}$ & $0.00 \pm 0.00 \mathrm{~d}$ & $1.10 \pm 0.10 \mathrm{a}$ & $25.25 \pm 0.25 \mathrm{e}$ \\
\hline & Ebonyi & $1.88 \pm 0.02 \mathrm{a}$ & $2.29 \pm 0.02 \mathrm{~b}$ & $1.74 \pm 0.07 \mathrm{c}$ & $0.00 \pm 0.00 \mathrm{~d}$ & $0.00 \pm 0.00$ & $0.07 \pm 0.01 \mathrm{~d}$ & $0.00 \pm 0.00 \mathrm{~d}$ & $0.17 \pm 0.01 \mathrm{~d}$ & $0.16 \pm 0.01 \mathrm{~d}$ & $0.01 \pm 0.00 \mathrm{~d}$ & $0.01 \pm 0.00 \mathrm{~d}$ & $4.95 \pm 0.05 \mathrm{e}$ \\
\hline & Enugu & $0.28 \pm 0.02 \mathrm{a}$ & $0.88 \pm 0.02 \mathrm{~b}$ & $0.03 \pm 0.02 \mathrm{c}$ & $0.11 \pm 0.01 \mathrm{~d}$ & $0.09 \pm 0.02 \mathrm{c}$ & $0.45 \pm 0.00 \mathrm{a}$ & $0.00 \pm 0.00 \mathrm{c}$ & $0.00 \pm 0.00 \mathrm{c}$ & $0.09 \pm 0.02 \mathrm{c}$ & $0.00 \pm 0.00 \mathrm{c}$ & $0.00 \pm 0.00 \mathrm{c}$ & $11.51 \pm 0.01 \mathrm{~d}$ \\
\hline \multirow[t]{2}{*}{ PYR } & Abia & $0.98 \pm 0.03 \mathrm{a}$ & $0.43 \pm 0.08 \mathrm{~b}$ & $0.29 \pm 0.01 \mathrm{c}$ & $0.10 \pm 0.00 \mathrm{~d}$ & $0.00 \pm 0.00 \mathrm{e}$ & $0.00 \pm 0.00 \mathrm{e}$ & $2.39 \pm 0.02 \mathrm{f}$ & $1.88 \pm 0.03 \mathrm{~g}$ & $0.00 \pm 0.00 \mathrm{e}$ & $0.00 \pm 0.00 \mathrm{e}$ & $0.00 \pm 0.00 \mathrm{e}$ & $0.06 \pm 0.01 \mathrm{~d}$ \\
\hline & Owerri & $0.88 \pm 0.03 a$ & $0.60 \pm 0.01 \mathrm{~b}$ & $0.10 \pm 0.01 \mathrm{c}$ & $0.00 \pm 0.00 \mathrm{~d}$ & $0.00 \pm 0.00 \mathrm{~d}$ & $0.00 \pm 0.00 \mathrm{~d}$ & $0.21 \pm 0.01 \mathrm{e}$ & $0.16 \pm 0.01 \mathrm{e}$ & $0.18 \pm 0.02 \mathrm{e}$ & $0.03 \pm 0.00 \mathrm{~d}$ & $0.00 \pm 0.00 \mathrm{~d}$ & $0.24 \pm 0.02 \mathrm{e}$ \\
\hline
\end{tabular}

Table 1. Mean concentration of PAHs (mg/kg dry weight) in crops from the study areas. The results are expressed as triplicate mean \pm S.E. 
Onyedikachi et al. / Ovidius University Annals of Chemistry 30 (2019) 37 - 43

\begin{tabular}{|c|c|c|c|c|c|c|c|c|c|c|c|c|c|}
\hline & & $\mathrm{VA}$ & TO & TT & ME & DR & $\mathrm{CA}$ & $\mathrm{CS}$ & CHA & $\mathbf{C P}$ & $\mathrm{CN}$ & $\mathrm{CO}$ & EG \\
\hline \multirow{7}{*}{ BaA } & Anambra & $4.95 \pm 0.02 \mathrm{a}$ & $0.20 \pm 0.01 \mathrm{~b}$ & $1.22 \pm 0.01 \mathrm{c}$ & $0.20 \pm 0.01 \mathrm{~d}$ & $0.10 \pm 0.01 \mathrm{~b}$ & $0.59 \pm 0.01 \mathrm{~d}$ & $0.00 \pm 0.00 \mathrm{e}$ & $0.10 \pm 0.01 \mathrm{~b}$ & $0.00 \pm 0.00 \mathrm{e}$ & $0.00 \pm 0.00 \mathrm{e}$ & $0.09 \pm 0.01 \mathrm{~b}$ & $2.91 \pm 0.10 \mathrm{f}$ \\
\hline & Ebonyi & $1.70 \pm 0.01 \mathrm{a}$ & $0.54 \pm 0.02 \mathrm{~b}$ & $1.28 \pm 0.02 \mathrm{c}$ & $0.00 \pm 0.00 \mathrm{~d}$ & $0.00 \pm 0.00 \mathrm{~d}$ & $1.41 \pm 0.09 \mathrm{a}$ & $0.00 \pm 0.00 \mathrm{~d}$ & $0.11 \pm 0.00 \mathrm{f}$ & $0.12 \pm 0.00 \mathrm{f}$ & $0.06 \pm 0.01 \mathrm{~d}$ & $0.02 \pm 0.01 \mathrm{~d}$ & $0.48 \pm 0.01 \mathrm{~b}$ \\
\hline & Enugu & $1.05 \pm 0.01 \mathrm{a}$ & $0.55 \pm 0.01 \mathrm{~b}$ & $0.05 \pm 0.00 \mathrm{c}$ & $0.11 \pm 0.00 \mathrm{~d}$ & $0.09 \pm 0.01 \mathrm{c}$ & $0.12 \pm 0.01 \mathrm{~d}$ & $0.00 \pm 0.00 \mathrm{e}$ & $0.00 \pm 0.00 \mathrm{e}$ & $0.17 \pm 0.01 \mathrm{~d}$ & $0.00 \pm 0.00 \mathrm{e}$ & $0.00 \pm 0.00 \mathrm{e}$ & $1.10 \pm 0.01 \mathrm{a}$ \\
\hline & Abia & $1.74 \pm 0.01 \mathrm{a}$ & $1.19 \pm 0.02 \mathrm{~b}$ & $0.54 \pm 0.03 \mathrm{c}$ & $0.03 \pm 0.00 \mathrm{~d}$ & $0.00 \pm 0.00 \mathrm{~d}$ & $0.00 \pm 0.00 \mathrm{~d}$ & $0.55 \pm 0.01 \mathrm{c}$ & $0.09 \pm 0.01 \mathrm{~d}$ & $0.00 \pm 0.00 \mathrm{~d}$ & $0.00 \pm 0.00 \mathrm{~d}$ & $0.00 \pm 0.00 \mathrm{~d}$ & $0.10 \pm 0.01 d$ \\
\hline & Owerri & $0.06 \pm 0.01 \mathrm{a}$ & $0.92 \pm 0.01 \mathrm{~b}$ & $1.55 \pm 0.04 \mathrm{c}$ & $0.00 \pm 0.00 \mathrm{~d}$ & $0.00 \pm 0.00 \mathrm{~d}$ & $0.00 \pm 0.00 \mathrm{~d}$ & $0.04 \pm 0.01 \mathrm{~d}$ & $0.29 \pm 0.02 \mathrm{~b}$ & $0.11 \pm 0.00 \mathrm{~b}$ & $0.02 \pm 0.01 \mathrm{~d}$ & $0.00 \pm 0.00 \mathrm{~d}$ & $0.35 \pm 0.00 \mathrm{~b}$ \\
\hline & Anambra & $8.94 \pm 0.01 \mathrm{a}$ & $0.72 \pm 0.01 \mathrm{~b}$ & $2.19 \pm 0.02 \mathrm{c}$ & $0.06 \pm 0.01 \mathrm{~d}$ & $0.02 \pm 0.01 \mathrm{~d}$ & $0.39 \pm 0.01 \mathrm{~b}$ & $0.00 \pm 0.00 \mathrm{e}$ & $0.18 \pm 0.01 \mathrm{~d}$ & $0.00 \pm 0.00 \mathrm{e}$ & $0.00 \pm 0.00 \mathrm{e}$ & $0.00 \pm 0.00 \mathrm{e}$ & $0.11 \pm 0.00 \mathrm{~d}$ \\
\hline & Ebonyi & $0.11 \pm 0.01 \mathrm{a}$ & $1.78 \pm 0.03 b$ & $2.28 \pm 0.02 \mathrm{c}$ & $0.00 \pm 0.00 \mathrm{~d}$ & $0.00 \pm 0.00 \mathrm{~d}$ & $0.06 \pm 0.00 \mathrm{~d}$ & $0.00 \pm 0.00 \mathrm{~d}$ & $0.00 \pm 0.00 \mathrm{~d}$ & $0.09 \pm 0.01 \mathrm{~d}$ & $0.02 \pm 0.00 \mathrm{~d}$ & $0.0 \pm 0.00 \mathrm{~d}$ & $0.78 \pm 0.04 \mathrm{e}$ \\
\hline \multirow{6}{*}{ CHR } & Enugu & $1.95 \pm 0.06 \mathrm{a}$ & $1.70 \pm 0.01 \mathrm{a}$ & $0.94 \pm 0.06 \mathrm{~b}$ & $0.03 \pm 0.00 \mathrm{c}$ & $0.02 \pm 0.01 \mathrm{c}$ & $0.15 \pm 0.01 \mathrm{~d}$ & $0.00 \pm 0.00 \mathrm{c}$ & $0.00 \pm 0.00 \mathrm{c}$ & $0.52 \pm 0.01 \mathrm{e}$ & $0.00 \pm 0.00 \mathrm{c}$ & $0.00 \pm 0.00 \mathrm{c}$ & $1.75 \pm 0.01 \mathrm{a}$ \\
\hline & Abia & $0.92 \pm 0.01 \mathrm{a}$ & $0.49 \pm 0.02 \mathrm{a}$ & $0.17 \pm 0.02 \mathrm{~b}$ & $0.01 \pm 0.00 \mathrm{c}$ & $0.00 \pm 0.00 \mathrm{c}$ & $0.00 \pm 0.00 \mathrm{c}$ & $1.16 \pm 0.01 \mathrm{~d}$ & $0.18 \pm 0.01 \mathrm{~b}$ & $0.00 \pm 0.00 \mathrm{c}$ & $0.00 \pm 0.00 \mathrm{c}$ & $0.00 \pm 0.00 \mathrm{c}$ & $0.02 \pm 0.00 \mathrm{c}$ \\
\hline & Owerri & $0.04 \pm 0.01 \mathrm{a}$ & $0.49 \pm 0.02 \mathrm{~b}$ & $3.61 \pm 0.02 \mathrm{c}$ & $0.00 \pm 0.00 \mathrm{~d}$ & $0.00 \pm 0.00 \mathrm{~d}$ & $0.00 \pm 0.00 \mathrm{~d}$ & $0.05 \pm 0.01 \mathrm{a}$ & $0.02 \pm 0.00 \mathrm{a}$ & $0.05 \pm 0.00 \mathrm{a}$ & $0.00 \pm 0.00 \mathrm{~d}$ & $0.00 \pm 0.00 \mathrm{~d}$ & $0.21 \pm 0.01 \mathrm{~b}$ \\
\hline & Anambra & $4.74 \pm 0.02 \mathrm{a}$ & $1.02 \pm 0.01 \mathrm{~b}$ & $0.65 \pm 0.01 \mathrm{c}$ & $0.02 \pm 0.00 \mathrm{~d}$ & $0.00 \pm 0.00 \mathrm{~d}$ & $0.20 \pm 0.01 \mathrm{e}$ & $0.00 \pm 0.00 \mathrm{~d}$ & $0.010 .00 \mathrm{~d}$ & $0.00 \pm 0.00 \mathrm{~d}$ & $0.00 \pm 0.00 \mathrm{~d}$ & $0.00 \pm 0.00 \mathrm{~d}$ & $0.04 \pm 0.01 \mathrm{~d}$ \\
\hline & Ebonyi & $0.06 \pm 0.01 \mathrm{a}$ & $0.71 \pm 0.01 \mathrm{~b}$ & $0.68 \pm 0.02 \mathrm{c}$ & $0.00 \pm 0.00 \mathrm{~d}$ & $0.00 \pm 0.00 \mathrm{~d}$ & $0.05 \pm 0.01 \mathrm{~d}$ & $0.00 \pm 0.00 \mathrm{~d}$ & $0.02 \pm 0.01 \mathrm{~d}$ & $0.05 \pm 0.01 \mathrm{~d}$ & $0.00 \pm 0.00 \mathrm{~d}$ & $0.00 \pm 0.00 \mathrm{~d}$ & $0.47 \pm 0.04 c$ \\
\hline & Enugu & $1.10 \pm 0.10 \mathrm{a}$ & $1.89 \pm 0.02 \mathrm{a}$ & $2.09 \pm 0.01 \mathrm{~b}$ & $0.03 \pm 0.02 \mathrm{c}$ & $0.00 \pm 0.00 \mathrm{c}$ & $0.19 \pm 0.01 \mathrm{~d}$ & $0.00 \pm 0.00 \mathrm{c}$ & $0.00 \pm 0.00 \mathrm{c}$ & $0.02 \pm 0.01 \mathrm{c}$ & $0.00 \pm 0.00 \mathrm{c}$ & $0.20 \pm 0.00 \mathrm{~d}$ & $1.01 \pm 0.00 \mathrm{a}$ \\
\hline \multirow[t]{5}{*}{ BbF } & Abia & $0.52 \pm 0.01 \mathrm{a}$ & $0.20 \pm 0.01 \mathrm{~b}$ & $0.19 \pm 0.01 \mathrm{~b}$ & $0.08 \pm 0.00 \mathrm{c}$ & $0.00 \pm 0.00 \mathrm{~d}$ & $0.00 \pm 0.00 \mathrm{~d}$ & $0.24 \pm 0.01 \mathrm{~b}$ & $0.48 \pm 0.02 \mathrm{a}$ & $0.00 \pm 0.00 \mathrm{~d}$ & $0.00 \pm 0.00 \mathrm{~d}$ & $0.01 \pm 0.00 \mathrm{~d}$ & $0.17 \pm 0.01 \mathrm{~b}$ \\
\hline & Owerri & $0.79 \pm 0.01 \mathrm{a}$ & $1.02 \pm 0.00 \mathrm{~b}$ & $0.03 \pm 0.00 \mathrm{c}$ & $0.00 \pm 0.00 \mathrm{c}$ & $0.00 \pm 0.00 \mathrm{c}$ & $0.00 \pm 0.00 \mathrm{c}$ & $0.09 \pm 0.02 \mathrm{~b}$ & $0.06 \pm 0.01 \mathrm{c}$ & $0.11 \pm 0.00 \mathrm{~b}$ & $0.23 \pm 0.01 \mathrm{~d}$ & $0.00 \pm 0.00 \mathrm{c}$ & $0.43 \pm 0.02 \mathrm{a}$ \\
\hline & Anambra & $2.64 \pm 0.01 \mathrm{a}$ & $9.34 \pm 0.01 \mathrm{~b}$ & $0.80 \pm 0.01 \mathrm{c}$ & $0.16 \pm 0.00 \mathrm{~d}$ & $0.06 \pm 0.01 \mathrm{e}$ & $0.09 \pm 0.01 \mathrm{e}$ & $0.00 \pm 0.00 \mathrm{f}$ & $0.03 \pm 0.01 \mathrm{f}$ & $0.00 \pm 0.00 \mathrm{f}$ & $0.00 \pm 0.00 \mathrm{f}$ & $0.00 \pm 0.00 \mathrm{f}$ & $0.29 \pm 0.02 \mathrm{~d}$ \\
\hline & Ebonyi & $1.55 \pm 0.01 \mathrm{a}$ & $0.30 \pm 0.01 \mathrm{~b}$ & $0.84 \pm 0.02 \mathrm{c}$ & $0.00 \pm 0.00 \mathrm{~d}$ & $0.00 \pm 0.00 \mathrm{~d}$ & $0.04 \pm 0.00 \mathrm{~d}$ & $0.00 \pm 0.00 \mathrm{~d}$ & $0.04 \pm 0.01 \mathrm{~d}$ & $0.09 \pm 0.01 \mathrm{e}$ & $0.51 \pm 0.01 \mathrm{c}$ & $0.00 \pm 0.00 \mathrm{~d}$ & $0.90 \pm 0.01 \mathrm{a}$ \\
\hline & Enugu & $0.56 \pm 0.00 \mathrm{a}$ & $10.61 \pm 0.01 \mathrm{~b}$ & $0.04 \pm 0.02 \mathrm{c}$ & $0.10 \pm 0.02 \mathrm{~d}$ & $0.05 \pm 0.01 \mathrm{c}$ & $0.48 \pm 0.02 \mathrm{a}$ & $0.00 \pm 0.00 \mathrm{c}$ & $0.00 \pm 0.00 \mathrm{c}$ & $0.02 \pm 0.01 \mathrm{c}$ & $0.00 \pm 0.00 \mathrm{c}$ & $0.09 \pm 0.00 \mathrm{c}$ & $2.09 \pm 0.02 \mathrm{e}$ \\
\hline \multirow[t]{5}{*}{ BkF } & Abia & $0.06 \pm 0.01 \mathrm{a}$ & $0.04 \pm 0.01 \mathrm{a}$ & $0.54 \pm 0.46 \mathrm{~b}$ & $0.04 \pm 0.00 \mathrm{a}$ & $0.00 \pm 0.00 \mathrm{a}$ & $0.00 \pm 0.00 \mathrm{a}$ & $0.17 \pm 0.01 \mathrm{c}$ & $0.04 \pm 0.00 \mathrm{a}$ & $0.00 \pm 0.00 \mathrm{a}$ & $0.00 \pm 0.00 \mathrm{a}$ & $0.02 \pm 0.00 \mathrm{a}$ & $0.09 \pm 0.01 \mathrm{a}$ \\
\hline & Owerri & $0.12 \pm 0.01 \mathrm{a}$ & $0.11 \pm 0.01 \mathrm{a}$ & $0.02 \pm 0.00 \mathrm{~b}$ & $0.00 \pm 0.00 \mathrm{c}$ & $0.00 \pm 0.00 \mathrm{c}$ & $0.00 \pm 0.00 \mathrm{c}$ & $0.04 \pm 0.00 \mathrm{~b}$ & $0.02 \pm 0.01 \mathrm{~b}$ & $0.05 \pm 0.02 \mathrm{~b}$ & $0.04 \pm 0.02 \mathrm{~b}$ & $0.00 \pm 0.00 \mathrm{c}$ & $0.06 \pm 0.01 \mathrm{~b}$ \\
\hline & Anambra & $0.29 \pm 0.01 \mathrm{a}$ & $2.48 \pm 0.03 b$ & $0.38 \pm 0.02 c$ & $0.09 \pm 0.01 \mathrm{~d}$ & $0.00 \pm 0.00 \mathrm{e}$ & $0.10 \pm 0.01 \mathrm{f}$ & $0.00 \pm 0.00 \mathrm{e}$ & $0.01 \pm 0.00 \mathrm{e}$ & $0.00 \pm 0.00 \mathrm{e}$ & $0.00 \pm 0.00 \mathrm{e}$ & $0.00 \pm 0.00 \mathrm{e}$ & $0.14 \pm 0.01 \mathrm{f}$ \\
\hline & Ebonyi & $0.23 \pm 0.01 \mathrm{a}$ & $0.07 \pm 0.02 \mathrm{~b}$ & $0.39 \pm 0.01 \mathrm{c}$ & $0.00 \pm 0.00 \mathrm{~d}$ & $0.00 \pm 0.00 \mathrm{~d}$ & $0.00 \pm 0.00 \mathrm{~d}$ & $0.00 \pm 0.00 \mathrm{~d}$ & $0.00 \pm 0.00 \mathrm{~d}$ & $0.03 \pm 0.01 \mathrm{~d}$ & $0.03 \pm 0.01 \mathrm{~d}$ & $0.00 \pm 0.01 \mathrm{~d}$ & $0.13 \pm 0.02 \mathrm{e}$ \\
\hline & Enugu & $0.07 \pm 0.01 \mathrm{a}$ & $1.29 \pm 0.07 \mathrm{~b}$ & $0.02 \pm 0.01 \mathrm{a}$ & $0.07 \pm 0.02 \mathrm{a}$ & $0.00 \pm 0.00 \mathrm{~d}$ & $0.06 \pm 0.00 \mathrm{~d}$ & $0.00 \pm 0.00 \mathrm{~d}$ & $0.00 \pm 0.00 \mathrm{~d}$ & $0.10 \pm 0.01 \mathrm{a}$ & $0.00 \pm 0.00 \mathrm{~d}$ & $0.05 \pm 0.00 \mathrm{~d}$ & $0.27 \pm 0.01 \mathrm{e}$ \\
\hline \multirow[t]{5}{*}{ BaP } & Abia & $0.00 \pm 0.00 \mathrm{a}$ & $0.02 \pm 0.01 \mathrm{a}$ & $0.15 \pm 0.01 \mathrm{~b}$ & $0.01 \pm 0.00 \mathrm{a}$ & $0.00 \pm 0.00 \mathrm{a}$ & $0.00 \pm 0.00 \mathrm{a}$ & $0.09 \pm 0.01 \mathrm{~b}$ & $0.20 \pm 0.00 \mathrm{c}$ & $0.00 \pm 0.00 \mathrm{a}$ & $0.00 \pm 0.00 \mathrm{a}$ & $0.00 \pm 0.00 \mathrm{a}$ & $0.01 \pm 0.00 \mathrm{a}$ \\
\hline & Owerri & $0.22 \pm 0.01 \mathrm{a}$ & $0.06 \pm 0.01 \mathrm{~b}$ & $0.05 \pm 0.00 \mathrm{~b}$ & $0.00 \pm 0.00 \mathrm{c}$ & $0.00 \pm 0.00 \mathrm{c}$ & $0.00 \pm 0.00 \mathrm{c}$ & $0.05 \pm 0.01 \mathrm{~b}$ & $0.07 \pm 0.01 \mathrm{~b}$ & $0.01 \pm 0.00 \mathrm{c}$ & $0.05 \pm 0.01 \mathrm{~b}$ & $0.00 \pm 0.00 \mathrm{c}$ & $0.39 \pm 0.02 \mathrm{~d}$ \\
\hline & Anambra & $0.02 \pm 0.00 \mathrm{a}$ & $3.38 \pm 0.02 b$ & $0.63 \pm 0.02 \mathrm{c}$ & $0.02 \pm 0.01 \mathrm{~d}$ & $0.03 \pm 0.01 \mathrm{e}$ & $0.05 \pm 0.01 \mathrm{f}$ & $0.00 \pm 0.00 \mathrm{c}$ & $0.05 \pm 0.01 \mathrm{f}$ & $0.00 \pm 0.00 \mathrm{a}$ & $0.00 \pm 0.00 \mathrm{a}$ & $0.00 \pm 0.01 \mathrm{a}$ & $0.17 \pm 0.01 \mathrm{~g}$ \\
\hline & Ebonyi & $0.44 \pm 0.02 \mathrm{a}$ & $0.03 \pm 0.01 \mathrm{~b}$ & $0.67 \pm 0.04 \mathrm{c}$ & $0.00 \pm 0.00 \mathrm{~d}$ & $0.00 \pm 0.00 \mathrm{~d}$ & $0.02 \pm 0.01 \mathrm{~d}$ & $0.00 \pm 0.00 \mathrm{~d}$ & $0.02 \pm 0.01 \mathrm{~d}$ & $0.02 \pm 0.01 \mathrm{~d}$ & $0.09 \pm 0.01 \mathrm{~d}$ & $0.00 \pm 0.00 \mathrm{~d}$ & $0.80 \pm 0.01 \mathrm{c}$ \\
\hline & Enugu & $0.00 \pm 0.00 \mathrm{a}$ & $2.41 \pm 0.01 \mathrm{~b}$ & $0.03 \pm 0.00 \mathrm{a}$ & $0.02 \pm 0.00 \mathrm{a}$ & $0.01 \pm 0.00 \mathrm{a}$ & $0.09 \pm 0.01 \mathrm{c}$ & $0.00 \pm 0.00 \mathrm{a}$ & $0.00 \pm 0.00 \mathrm{a}$ & $0.08 \pm 0.02 \mathrm{c}$ & $0.00 \pm 0.00 \mathrm{a}$ & $0.01 \pm 0.00 \mathrm{a}$ & $1.84 \pm 0.00 \mathrm{~d}$ \\
\hline \multirow[t]{5}{*}{ IcdP } & Abia & $0.04 \pm 0.01 \mathrm{a}$ & $0.14 \pm 0.02 \mathrm{~b}$ & $0.91 \pm 0.09 \mathrm{c}$ & $0.08 \pm 0.00 \mathrm{~d}$ & $0.00 \pm 0.00 \mathrm{e}$ & $0.00 \pm 0.00 \mathrm{e}$ & $0.54 \pm 0.01 \mathrm{c}$ & $1.48 \pm 0.02 \mathrm{f}$ & $0.00 \pm 0.00 \mathrm{e}$ & $0.00 \pm 0.00 \mathrm{e}$ & $0.01 \pm 0.00 \mathrm{e}$ & $0.81 \pm 0.02 \mathrm{c}$ \\
\hline & Owerri & $0.19 \pm 0.01 \mathrm{a}$ & $0.35 \pm 0.01 \mathrm{~b}$ & $0.05 \pm 0.02 \mathrm{c}$ & $0.00 \pm 0.00 \mathrm{~d}$ & $0.00 \pm 0.00 \mathrm{~d}$ & $0.00 \pm 0.00 \mathrm{~d}$ & $0.05 \pm 0.01 \mathrm{c}$ & $0.05 \pm 0.01 \mathrm{c}$ & $0.02 \pm 0.00 \mathrm{c}$ & $0.01 \pm 0.00 \mathrm{~d}$ & $0.00 \pm 0.00 \mathrm{~d}$ & $0.00 \pm 0.00 \mathrm{~d}$ \\
\hline & Anambra & $0.17 \pm 0.01 \mathrm{a}$ & $1.47 \pm 0.03 \mathrm{~b}$ & $3.51 \pm 0.01 \mathrm{c}$ & $0.16 \pm 0.01 \mathrm{a}$ & $0.00 \pm 0.00 \mathrm{~d}$ & $0.21 \pm 0.01 \mathrm{a}$ & $0.00 \pm 0.00 \mathrm{~d}$ & $0.00 \pm 0.00 \mathrm{~d}$ & $0.00 \pm 0.00 \mathrm{~d}$ & $0.00 \pm 0.00 \mathrm{~d}$ & $0.00 \pm 0.01 \mathrm{~d}$ & $0.22 \pm 0.01 \mathrm{a}$ \\
\hline & Ebonyi & $0.38 \pm 0.02 \mathrm{a}$ & $0.19 \pm 0.01 \mathrm{~b}$ & $3.68 \pm 0.02 c$ & $0.00 \pm 0.00 \mathrm{~d}$ & $0.00 \pm 0.00 \mathrm{~d}$ & $0.02 \pm 0.00 \mathrm{~d}$ & $0.00 \pm 0.00 \mathrm{~d}$ & $0.09 \pm 0.01 \mathrm{~b}$ & $0.02 \pm 0.00 \mathrm{~d}$ & $0.03 \pm 0.00$ & $0.00 \pm 0.00 \mathrm{~d}$ & $0.00 \pm 0.00 \mathrm{~d}$ \\
\hline & Enugu & $0.04 \pm 0.01 \mathrm{a}$ & $0.07 \pm 0.01 \mathrm{a}$ & $0.02 \pm 0.00 \mathrm{a}$ & $0.09 \pm 0.00 \mathrm{a}$ & $0.00 \pm 0.00 \mathrm{~b}$ & $0.02 \pm 0.00 \mathrm{a}$ & $0.00 \pm 0.00 \mathrm{~b}$ & $0.00 \pm 0.00 \mathrm{~b}$ & $0.04 \pm 0.01 \mathrm{a}$ & $0.00 \pm 0.00 \mathrm{~b}$ & $0.03 \pm 0.00 \mathrm{a}$ & $0.01 \pm 0.00 \mathrm{a}$ \\
\hline Dah & Abia & $0.09 \pm 0.02 \mathrm{a}$ & $0.21 \pm 0.01 \mathrm{~b}$ & $0.55 \pm 0.03 \mathrm{c}$ & $0.30 \pm 0.00 \mathrm{~b}$ & $0.00 \pm 0.00 \mathrm{~d}$ & $0.00 \pm 0.00 \mathrm{~d}$ & $0.20 \pm 0.01 \mathrm{~b}$ & $0.54 \pm 0.01 \mathrm{c}$ & $0.00 \pm 0.00 \mathrm{~d}$ & $0.00 \pm 0.00 \mathrm{~d}$ & $0.01 \pm 0.00 \mathrm{~d}$ & $4.33 \pm 0.02 \mathrm{e}$ \\
\hline \multirow[t]{4}{*}{ A } & Owerri & $0.46 \pm 0.01 \mathrm{a}$ & $1.31 \pm 0.01 \mathrm{~b}$ & $0.39 \pm 0.02 \mathrm{a}$ & $0.00 \pm 0.00 \mathrm{c}$ & $0.00 \pm 0.00 \mathrm{c}$ & $0.00 \pm 0.00 \mathrm{c}$ & $0.00 \pm 0.00 \mathrm{c}$ & $0.02 \pm 0.00 \mathrm{c}$ & $0.15 \pm 0.02 \mathrm{~d}$ & $0.05 \pm 0.00 \mathrm{c}$ & $0.00 \pm 0.00 \mathrm{c}$ & $0.23 \pm 0.00 \mathrm{a}$ \\
\hline & Anambra & $0.39 \pm 0.01 \mathrm{a}$ & $10.21 \pm 0.01 \mathrm{~b}$ & $2.37 \pm 0.14 c$ & $0.62 \pm 0.00 \mathrm{a}$ & $0.32 \pm 0.01 \mathrm{a}$ & $0.16 \pm 0.01 \mathrm{~d}$ & $0.00 \pm 0.00 \mathrm{e}$ & $0.01 \pm 0.00 \mathrm{e}$ & $0.00 \pm 0.00 \mathrm{e}$ & $0.00 \pm 0.00 \mathrm{e}$ & $0.00 \pm 0.00 \mathrm{e}$ & $0.40 \pm 0.01 \mathrm{a}$ \\
\hline & Ebonyi & $0.95 \pm 0.05 \mathrm{a}$ & $0.39 \pm 0.01 \mathrm{~b}$ & $2.34 \pm 0.02 \mathrm{c}$ & $0.00 \pm 0.00 \mathrm{~d}$ & $0.00 \pm 0.00 \mathrm{~d}$ & $0.03 \pm 0.01 \mathrm{~d}$ & $0.00 \pm 0.00 \mathrm{~d}$ & $0.04 \pm 0.01 \mathrm{~d}$ & $0.10 \pm 0.00 \mathrm{~d}$ & $0.10 \pm 0.00 \mathrm{~d}$ & $0.00 \pm 0.01 \mathrm{~d}$ & $0.50 \pm 0.01 \mathrm{~d}$ \\
\hline & Enugu & $0.09 \pm 0.01 \mathrm{a}$ & $0.19 \pm 0.02 \mathrm{~b}$ & $0.22 \pm 0.01 \mathrm{~b}$ & $0.38 \pm 0.02 \mathrm{~b}$ & $0.29 \pm 0.02 \mathrm{~b}$ & $0.57 \pm 0.03 \mathrm{c}$ & $0.00 \pm 0.00 \mathrm{~d}$ & $0.00 \pm 0.00 \mathrm{~d}$ & $0.05 \pm 0.00 \mathrm{~d}$ & $0.00 \pm 0.00 \mathrm{~d}$ & $0.00 \pm 0.00 \mathrm{~d}$ & $1.18 \pm 0.03 \mathrm{e}$ \\
\hline
\end{tabular}

Values in different letters in the same column are significantly different at 0.05 level $(p \leq 0.05)$ while same letters in the same column are not significantly different $(p \leq 0.05)$. Values $(0.00 \mathrm{mg} / \mathrm{kg})$ are BDL - below detection limit of $0.001 \mathrm{mg} / \mathrm{kg}$.

Table 2. Daily intakes of PAHs (mg kg body weight per day) for adults and children.

\begin{tabular}{|c|c|c|c|c|c|c|c|c|c|c|c|c|c|}
\hline Location & & VA & TO & TT & ME & DR & CA & CS & CHA & $\mathbf{C P}$ & $\mathrm{CN}$ & $\mathrm{CO}$ & EG \\
\hline \multirow[t]{2}{*}{ Osisioma } & Adults & $2.42 \mathrm{E}-01$ & $4.34 \mathrm{E}-01$ & $1.08 \mathrm{E}+00$ & $6.42 \mathrm{E}-01$ & $0.00 \mathrm{E}+00$ & $0.00 \mathrm{E}+00$ & $4.36 \mathrm{E}-01$ & $1.08 \mathrm{E}+00$ & $0.00 \mathrm{E}+00$ & $0.00 \mathrm{E}+00$ & $1.86 \mathrm{E}-02$ & $7.51 \mathrm{E}+00$ \\
\hline & Children & $1.47 \mathrm{E}-01$ & 2.63E-01 & $6.54 \mathrm{E}-01$ & $3.56 \mathrm{E}-01$ & $0.00 \mathrm{E}+00$ & $0.00 \mathrm{E}+00$ & $2.64 \mathrm{E}-01$ & $6.53 \mathrm{E}-01$ & $0.00 \mathrm{E}+00$ & $0.00 \mathrm{E}+00$ & $1.13 \mathrm{E}-02$ & $4.55 \mathrm{E}+00$ \\
\hline \multirow[t]{2}{*}{ Akwuuru } & Adults & $1.12 \mathrm{E}+00$ & $1.93 \mathrm{E}+01$ & $4.56 \mathrm{E}+00$ & $1.33 \mathrm{E}+00$ & $6.85 \mathrm{E}-01$ & $3.90 \mathrm{E}-01$ & $0.00 \mathrm{E}+00$ & $4.25 \mathrm{E}-02$ & $0.00 \mathrm{E}+00$ & $0.00 \mathrm{E}+00$ & $5.76 \mathrm{E}-04$ & $7.85 \mathrm{E}-01$ \\
\hline & Children & $6.80 \mathrm{E}-01$ & $1.17 \mathrm{E}+01$ & $2.76 \mathrm{E}+00$ & 7.36E-01 & $3.80 \mathrm{E}-01$ & $2.17 \mathrm{E}-01$ & $0.00 \mathrm{E}+00$ & $2.58 \mathrm{E}-02$ & $0.00 \mathrm{E}+00$ & $0.00 \mathrm{E}+00$ & $3.49 \mathrm{E}-04$ & $4.76 \mathrm{E}-01$ \\
\hline \multirow[t]{2}{*}{ Irete } & Adults & $9.12 \mathrm{E}-01$ & $2.37 \mathrm{E}+00$ & $7.60 \mathrm{E}-01$ & $0.00 \mathrm{E}+00$ & $0.00 \mathrm{E}+00$ & $0.00 \mathrm{E}+00$ & $2.56 \mathrm{E}-02$ & $7.38 \mathrm{E}-02$ & $2.73 \mathrm{E}-01$ & $1.14 \mathrm{E}-01$ & $0.00 \mathrm{E}+00$ & $5.62 \mathrm{E}-01$ \\
\hline & Children & $5.52 \mathrm{E}-01$ & $1.43 \mathrm{E}+00$ & $4.60 \mathrm{E}-01$ & $0.00 \mathrm{E}+00$ & $0.00 \mathrm{E}+00$ & $0.00 \mathrm{E}+00$ & $1.55 \mathrm{E}-02$ & $4.47 \mathrm{E}-02$ & $1.65 \mathrm{E}-01$ & $6.91 \mathrm{E}-02$ & $0.00 \mathrm{E}+00$ & $3.41 \mathrm{E}-01$ \\
\hline \multirow[t]{2}{*}{ Ishiagu } & Adults & $1.87 \mathrm{E}+00$ & $7.79 \mathrm{E}-01$ & $4.53 \mathrm{E}+00$ & $0.00 \mathrm{E}+00$ & $0.00 \mathrm{E}+00$ & $7.73 \mathrm{E}-02$ & $0.00 \mathrm{E}+00$ & $8.08 \mathrm{E}-02$ & $1.88 \mathrm{E}-01$ & $2.24 \mathrm{E}-01$ & $6.90 \mathrm{E}-05$ & $1.21 \mathrm{E}+00$ \\
\hline & Children & $1.13 \mathrm{E}+00$ & $4.72 \mathrm{E}-01$ & $2.74 \mathrm{E}+00$ & $0.00 \mathrm{E}+00$ & $0.00 \mathrm{E}+00$ & $4.29 \mathrm{E}-02$ & $0.00 \mathrm{E}+00$ & $4.90 \mathrm{E}-02$ & $1.14 \mathrm{E}-01$ & $1.36 \mathrm{E}-01$ & 4.18E-05 & $7.30 \mathrm{E}-01$ \\
\hline Ngwo & Adults & $2.52 \mathrm{E}-01$ & $1.64 \mathrm{E}+00$ & $4.32 \mathrm{E}-01$ & $8.16 \mathrm{E}-01$ & $6.13 \mathrm{E}-01$ & $1.26 \mathrm{E}+00$ & $0.00 \mathrm{E}+00$ & $0.00 \mathrm{E}+00$ & $1.38 \mathrm{E}-01$ & $0.00 \mathrm{E}+00$ & $1.00 \mathrm{E}-02$ & $2.82 \mathrm{E}+00$ \\
\hline
\end{tabular}

Table 3. Incremental life time cancer risk for adults and children using the total observed TEBaP concentration for each food crop.

\begin{tabular}{ccccccccccccccccc}
\hline Location & & VA & TO & TT & ME & DR & CA & CS & CHA & CP & CN & CO & EG \\
\hline Osisioma & Adults & $2.94 \mathrm{E}-08$ & $5.28 \mathrm{E}-08$ & $1.31 \mathrm{E}-07$ & $7.81 \mathrm{E}-08$ & $0.00 \mathrm{E}+00$ & $0.00 \mathrm{E}+00$ & $5.30 \mathrm{E}-08$ & $1.31 \mathrm{E}-07$ & $0.00 \mathrm{E}+00$ & $0.00 \mathrm{E}+00$ & $2.26 \mathrm{E}-09$ & $9.14 \mathrm{E}-07$ \\
& Children & $3.28 \mathrm{E}-08$ & $5.87 \mathrm{E}-08$ & $1.46 \mathrm{E}-07$ & $7.95 \mathrm{E}-08$ & $0.00 \mathrm{E}+00$ & $0.00 \mathrm{E}+00$ & $5.89 \mathrm{E}-08$ & $1.46 \mathrm{E}-07$ & $0.00 \mathrm{E}+00$ & $0.00 \mathrm{E}+00$ & $2.52 \mathrm{E}-09$ & $1.02 \mathrm{E}-06$ \\
\multirow{5}{*}{ Akwuuru } & Adults & $1.36 \mathrm{E}-07$ & $2.35 \mathrm{E}-06$ & $5.55 \mathrm{E}-07$ & $1.62 \mathrm{E}-07$ & $8.33 \mathrm{E}-08$ & $4.75 \mathrm{E}-08$ & $0.00 \mathrm{E}+00$ & $5.17 \mathrm{E}-09$ & $0.00 \mathrm{E}+00$ & $0.00 \mathrm{E}+00$ & $7.01 \mathrm{E}-11$ & $9.55 \mathrm{E}-08$ \\
& Children & $1.52 \mathrm{E}-07$ & $2.61 \mathrm{E}-06$ & $6.16 \mathrm{E}-07$ & $1.64 \mathrm{E}-07$ & $8.48 \mathrm{E}-08$ & $4.84 \mathrm{E}-08$ & $0.00 \mathrm{E}+00$ & $5.76 \mathrm{E}-09$ & $0.00 \mathrm{E}+00$ & $0.00 \mathrm{E}+00$ & $7.79 \mathrm{E}-11$ & $1.06 \mathrm{E}-07$ \\
\multirow{5}{*}{ Irete } & Adults & $1.11 \mathrm{E}-07$ & $2.88 \mathrm{E}-07$ & $9.25 \mathrm{E}-08$ & $0.00 \mathrm{E}+00$ & $0.00 \mathrm{E}+00$ & $0.00 \mathrm{E}+00$ & $3.11 \mathrm{E}-09$ & $8.98 \mathrm{E}-09$ & $3.32 \mathrm{E}-08$ & $1.39 \mathrm{E}-08$ & $0.00 \mathrm{E}+00$ & $6.84 \mathrm{E}-08$ \\
& Children & $1.23 \mathrm{E}-07$ & $3.19 \mathrm{E}-07$ & $1.03 \mathrm{E}-07$ & $0.00 \mathrm{E}+00$ & $0.00 \mathrm{E}+00$ & $0.00 \mathrm{E}+00$ & $3.46 \mathrm{E}-09$ & $9.98 \mathrm{E}-09$ & $3.68 \mathrm{E}-08$ & $1.54 \mathrm{E}-08$ & $0.00 \mathrm{E}+00$ & $7.61 \mathrm{E}-08$ \\
\multirow{3}{*}{ Ishiagu } & Adults & $2.28 \mathrm{E}-07$ & $9.48 \mathrm{E}-08$ & $5.51 \mathrm{E}-07$ & $0.00 \mathrm{E}+00$ & $0.00 \mathrm{E}+00$ & $9.40 \mathrm{E}-09$ & $0.00 \mathrm{E}+00$ & $9.83 \mathrm{E}-09$ & $2.29 \mathrm{E}-08$ & $2.73 \mathrm{E}-08$ & $8.40 \mathrm{E}-12$ & $1.47 \mathrm{E}-07$ \\
& Children & $2.52 \mathrm{E}-07$ & $1.05 \mathrm{E}-07$ & $6.12 \mathrm{E}-07$ & $0.00 \mathrm{E}+00$ & $0.00 \mathrm{E}+00$ & $9.58 \mathrm{E}-09$ & $0.00 \mathrm{E}+00$ & $1.09 \mathrm{E}-08$ & $2.54 \mathrm{E}-08$ & $3.04 \mathrm{E}-08$ & $9.33 \mathrm{E}-12$ & $1.63 \mathrm{E}-07$ \\
\multirow{2}{*}{ Ngwo } & Adults & $3.07 \mathrm{E}-08$ & $2.00 \mathrm{E}-07$ & $5.26 \mathrm{E}-08$ & $9.93 \mathrm{E}-08$ & $7.46 \mathrm{E}-08$ & $1.53 \mathrm{E}-07$ & $0.00 \mathrm{E}+00$ & $0.00 \mathrm{E}+00$ & $1.68 \mathrm{E}-08$ & $0.00 \mathrm{E}+00$ & $1.22 \mathrm{E}-09$ & $3.43 \mathrm{E}-07$ \\
& Children & $3.42 \mathrm{E}-08$ & $2.21 \mathrm{E}-07$ & $5.85 \mathrm{E}-08$ & $1.01 \mathrm{E}-07$ & $7.59 \mathrm{E}-08$ & $1.56 \mathrm{E}-07$ & $0.00 \mathrm{E}+00$ & $0.00 \mathrm{E}+00$ & $1.86 \mathrm{E}-08$ & $0.00 \mathrm{E}+00$ & $1.35 \mathrm{E}-09$ & $3.82 \mathrm{E}-07$ \\
& & $8.92 \mathrm{E}-08$ & $4.98 \mathrm{E}-07$ & $2.30 \mathrm{E}-07$ & $5.66 \mathrm{E}-08$ & $2.63 \mathrm{E}-08$ & $3.50 \mathrm{E}-08$ & $9.35 \mathrm{E}-09$ & $2.58 \mathrm{E}-08$ & $1.22 \mathrm{E}-08$ & $6.87 \mathrm{E}-09$ & $5.93 \mathrm{E}-10$ & $2.61 \mathrm{E}-07$ \\
\hline
\end{tabular}

Table 4. Total PAHs (mg/kg dry weight) and benzo(a)pyrene ( $\mathrm{mg} / \mathrm{kg}$ dry weight) concentration in food crops.

\begin{tabular}{|c|c|c|c|c|c|c|c|c|c|c|c|c|c|}
\hline Location & & VA & TO & TT & ME & DR & $\overline{\mathbf{C A}}$ & CS & CHA & $\mathbf{C P}$ & $\mathrm{CN}$ & $\mathrm{CO}$ & EG \\
\hline \multirow[t]{2}{*}{ Osisioma } & $\begin{array}{l}\text { Total } \\
\text { PAHs }\end{array}$ & 6.24 & 14.23 & 5.87 & 1.34 & 0.00 & 0.00 & 11.04 & 14.49 & 0.00 & 0.00 & 0.05 & 6.13 \\
\hline & $\Sigma \mathrm{BEC}$ & 7.02E-01 & $1.26 \mathrm{E}+00$ & $3.13 \mathrm{E}+00$ & $1.53 \mathrm{E}+00$ & $0.00 \mathrm{E}+00$ & $0.00 \mathrm{E}+00$ & $1.26 \mathrm{E}+00$ & $3.13 \mathrm{E}+00$ & $0.00 \mathrm{E}+00$ & $0.00 \mathrm{E}+00$ & $5.40 \mathrm{E}-02$ & $2.18 \mathrm{E}+01$ \\
\hline \multirow[t]{2}{*}{ Akwuuru } & $\begin{array}{l}\text { Total } \\
\text { PAHs }\end{array}$ & 31.66 & 36.29 & 22.74 & 2.8 & 1.00 & 2.37 & 0.00 & 0.89 & 0.00 & 0.00 & 1.58 & 30.13 \\
\hline & $\Sigma \mathrm{BEC}$ & $3.26 \mathrm{E}+00$ & $5.59 \mathrm{E}+01$ & $1.32 \mathrm{E}+01$ & $3.17 \mathrm{E}+00$ & $1.64 \mathrm{E}+00$ & $9.33 \mathrm{E}-01$ & $0.00 \mathrm{E}+00$ & $1.23 \mathrm{E}-01$ & $0.00 \mathrm{E}+00$ & $0.00 \mathrm{E}+00$ & $1.67 \mathrm{E}-03$ & $2.28 \mathrm{E}+00$ \\
\hline \multirow[t]{2}{*}{ Irete } & $\begin{array}{l}\text { Total } \\
\text { PAHs }\end{array}$ & 4.59 & 7.5 & 6.11 & 0.00 & 0.00 & 0.00 & 1.41 & 1.46 & 1.03 & 0.7 & 0.00 & 4.53 \\
\hline & $\Sigma \mathrm{BEC}$ & $2.64 \mathrm{E}+00$ & $6.86 \mathrm{E}+00$ & $2.20 \mathrm{E}+00$ & $0.00 \mathrm{E}+00$ & $0.00 \mathrm{E}+00$ & $0.00 \mathrm{E}+00$ & 7.42E-02 & $2.14 \mathrm{E}-01$ & $7.90 \mathrm{E}-01$ & $3.30 \mathrm{E}-01$ & $0.00 \mathrm{E}+00$ & $1.63 \mathrm{E}+00$ \\
\hline \multirow[t]{2}{*}{ Ishiagu } & $\begin{array}{l}\text { Total } \\
\text { PAHs }\end{array}$ & 8.94 & 20.98 & 23.36 & 0.00 & 0,00 & 2.03 & 0.00 & 0.95 & 0.79 & 1.42 & 0.11 & 9.5 \\
\hline & $\Sigma$ BEC & $5.43 \mathrm{E}+00$ & $2.26 \mathrm{E}+00$ & $1.31 \mathrm{E}+01$ & $0.00 \mathrm{E}+00$ & $0.00 \mathrm{E}+00$ & $1.85 \mathrm{E}-01$ & $0.00 \mathrm{E}+00$ & $2.34 \mathrm{E}-01$ & $5.44 \mathrm{E}-01$ & $6.50 \mathrm{E}-01$ & $2.00 \mathrm{E}-04$ & $3.49 \mathrm{E}+00$ \\
\hline \multirow[t]{2}{*}{ Ngwo } & $\begin{array}{l}\text { Total } \\
\text { PAHs }\end{array}$ & 6.95 & 1.97 & 3.61 & 1.7 & 8.7 & 4.39 & 0.00 & 0.00 & 1.25 & 0.00 & 3.8 & 2.18 \\
\hline & इBEC & 7.30E-01 & $4.75 \mathrm{E}+00$ & $1.25 \mathrm{E}+00$ & $1.95 \mathrm{E}+00$ & $1.47 \mathrm{E}+00$ & $3.02 \mathrm{E}+00$ & $0.00 \mathrm{E}+00$ & $0.00 \mathrm{E}+00$ & $3.99 \mathrm{E}-01$ & $0.00 \mathrm{E}+00$ & $2.90 \mathrm{E}-02$ & $8.18 \mathrm{E}+00$ \\
\hline
\end{tabular}


Mean concentration of PAHs in foods crops. Mean concentration of PAHs in food samples (Table 1) ranged from below detection limit $(<0.01)$ to $2.64 \pm 0.02,5.27$ $\pm 0.04,0.96 \pm 0.02,8.94 \pm 0.01$ and $1.95 \pm 0.06 \mathrm{mg} / \mathrm{kg}$ for Star apple collected from Osisioma, pumpkin leaf from Ishiagu, and bitter leaf from Irete, Akwuuru and Ngwo respectively. This may be attributable to the uptake of PAHs by plant through gaseous (aerial) and particle bound deposition $[15,16]$. These depicts serious environmental concerns which may be lethal to the entire populace. These high molecular weight PAHs are associated with prolonged effects due to less susceptibility to microbial degradation [6]. Consequently, the population of people living in and around the study sites may be predisposed to high risk of cancer due to long exposure to $\mathrm{PAH}$ compound through contaminated soil and food crops.

Total PAHs concentrations (Table 4) in most crop samples had highest values 14.49, 36.29, 4.59, 23.36 and $21.8 \mathrm{in} \mathrm{mg} / \mathrm{kg}$ for Chyrysophyllum albidum, Telferia occidentalis, Vernonia amygdalina, Talinum triangulare and Elaies guinnensis for Abia (Osisioma), Anambra (Akwuuru), Imo (Irete), Ebonyi (Ishiagu) and Enugu (Ngwo) respectively. These exceeded the European Union regulatory limit of $0.2 \mathrm{mg} / \mathrm{kg} \mathrm{d} . \mathrm{w}$. in foods suggesting possible health risk of exposure to PAHs through the food chain for the populace in these locals. Low IQ (intelligent quotient), untimely labor, low birth weight, asthma in children, DNA damages, cardiac malfunction etc. have been reported in children with high pre-natal exposure to PAHs [10, 17].

Soil-to-plant transfer is a major route of contamination of plants. It is also important to consider air deposits, PAHs volatilization from soil as well as the maturity and the age of the plant. The deposits of dust containing PAHs could be adsorbed on plant surface although PAHs can also be partly cleaned off during rainfall $[6,18]$. However, the relation between PAHs levels and maturity age has not been fully elucidated by authors [19]. Generally, comparison of PAHs distributions in food samples analyzed in this study has shown vegetables to be significant higher $(p \leq 0.05)$ than other crops. The PAHs distribution followed the order vegetables > fruits > nuts > tubers. Interestingly, vegetables from all the areas accumulated higher levels of PAHs significantly $(p \leq 0.05)$ compared to other classes of food crops analyzed. The abundance of PAHs in vegetables analyzed in this study may be due to their relatively high solubility, volatility and bioavailability $[20,21]$. It could also be attributed to their large surface area which is in constant contact with air laden with dust and pollutants like PAHs [16]. The distribution of PAHs in this study had higher concentrations in Vernonia amygdalina (a vegetable with broad and rough-surface leaves) more than the other two vegetables $T$. triangulare and $T$. occidentalis, vegetables often consumed by the Igbos. This agreed with Inam [22], where V. amygdelina had higher PAHs concentrations than in Lasianthera africanum, a vegetable described by Paris et al. [6], as a small and smooth leaved vegetable. The higher concentration present in $V$. amygdalina may be due to the adsorption of volatile organic compounds dependent on plant's morphology as a result of its larger surface of exchange with the gas phase which could increase plant contamination $[6,22]$.

On the other hand, fruits mainly are often consumed raw and this is particularly disturbing as there might be no loss at all in the actual concentration of PAHs in the fruit as they are ingested unprocessed directly into the alimentary canal. Other crop samples in this study are usually not consumed raw. Cooking is known way for preparation vegetable soup for the south east people in Nigeria, and this practice may decrease the total PAHs content in PAHs contaminated foods [23]. For instance, Paris et al. [6] reported that a considerable PAHs content was found in the water used to boil/cook potatoes indicating a possible transfer during the cooking process. This may also depend on the food species, temperature of heat source, oxygen availability, cooking time and the kind of cooking process. Roasting is also another process of food preparation in South Eastern Nigeria. This method is usually practiced for tubers like yam and this could also increase the PAHs content in plant foods [24]. However, cassava which is processed into a staple food consumed in South Eastern Nigeria (fufu) undergoes fermentation process during its preparation and thus may degrade PAHs content via enzymatic and microbial degradation.

Samples from all the industrial locations in this study had the summation of their 2-ringed PAHs (naphthalene and 2-methylnaphthalene) concentrations as follows $9.95,0.68,5.30,8.85$ and $0.84 \mathrm{mg} / \mathrm{kg}$. Also, 3-ringed PAHs i.e acenaphthylene, acenaphthene, fluorene, phenanthrene and anthracene gave values of 15.7, 3.51, $22.57,18.81$ and $5.77 \mathrm{mg} / \mathrm{kg}$ respectively as seen on table 1. The 4-ringed PAHs (fluoranthene, pyrene, benz(a) anthracene and chrysene) concentrations also were 20.13, $15.69,60.12,14.16$ and $30.27 \mathrm{mg} / \mathrm{kg}$, while the 5 -ringed PAHs (benzo(b) fluoranthene, benzo(k)fluoranthene, benzo(a) pyrene and dibenzo(ah)anthracene) 7.38, 4.84, $26.99,11.65$ and $20.78 \mathrm{mg} / \mathrm{kg}$ and the concentration of 56-ring PAHS (indeno (1,2,3-cd) pyrene) was 6.23, 2.61, $14.48,4.45$ and $2.97 \mathrm{mg} / \mathrm{kg} \mathrm{d}$.w. for all the food crops grown in the study areas at Osisioma, Irete, Akwuuru, Ishiagu and Ngwo respectively.

Low molecular weight PAHs from values obtained appears to be prevalent over the high molecular weight and thus can indicating serious cancer risk to human health [25]. PAHs ingested via food consumption can act in synergy with other carcinogens to escalate the proliferation of cancerous cells [25]. The accumulation of PAHs in foods could be as a result of the nearness of agricultural lands to the industrial/urban areas where high levels of anthropogenic activities occur [6]. Furthermore, differences in the concentration levels of PAHs generally could be as a result of differences in the bioaccumulation of plants and the proximity to the pollution source [10].

Human health risk assessment. Benzo(a)pyrene (BaP) in analyzed samples from study sites gave values above the DPR intervention limit of $0.01 \mathrm{mg} / \mathrm{kg}$ of $\mathrm{B}(\mathrm{a}) \mathrm{P}$ in some foods [10]. Samples had concentrations highest in Telferia occidentalis $(3.38 \pm 0.02 ; 2.41 \pm 0.01 \mathrm{mg} / \mathrm{kg})$ for Akwuuru and Ngwo followed by Elaies guineensis (1.84 $\pm 0.00 \mathrm{mg} / \mathrm{kg}$ ) suggesting their use as bio-monitoring 
plants. Citrus sinensis and Cola acuminata had B(a)P concentrations consistently below the DPR limits of 0.01 $\mathrm{mg} / \mathrm{kg}$ d.w. [11]. This may be because the part of plant analyzed was the endocarp (the part often consumed) of the plant. B(a)P seems to have preferentially accumulated in the peel as seen [19] which was not analyzed in this study. The lipid content of peels is generally higher than in the endocarp, this could describe the basis for increased levels of hydrophobic contaminants in the plant part $[6,26]$. B(a)P toxic equivalence to other PAHs is often ascertained that because it is the key PAHs congener monitored in highly consumed food products [6].

The B(a)P-equivalent (BEC) carcinogenic and mutagenic PAHs potency represents potential cancer and mutation effects evaluation are shown in Table 4. Results had highest values as follows $2.18 \mathrm{E}+01,1.31 \mathrm{E}+01$ for Talinum triangulare in Osisioma and Ishiagu respectively, $5.59 \mathrm{E}+01$ and $6.86 \mathrm{E}+01$ in Telferia occidentalis in Irete and Nnewi respectively, while highest BEC was seen in Elaies guineensis (8.18 E+01) in Ngwo. About $58.3 \%$ of $\Sigma$ BEC in food crops exceeded common regulatory target cleanup levels for PAHs and B(a)P-TE (0.1-0.66 mg/kg) as stated by Nwaichi et al. [10].

The Daily Dietary Intake (DDI) of PAHs via food consumption for the populace in this study was calculated (Table 2). DDI for adults were within the range of 0.00 $\mathrm{mg} / \mathrm{kg} /$ body to $7.51 \mathrm{E}+00,4.56 \mathrm{E}+00,2.37 \mathrm{E}+00,4.53$ $\mathrm{E}+00, \quad 2.82 \mathrm{E}+00 \mathrm{mg} / \mathrm{kg} /$ body (adults) and 0.00 $\mathrm{mg} / \mathrm{kg} / \mathrm{body}$ to $4.55 \mathrm{E}+00,2.76 \mathrm{E}+00,1.43 \mathrm{E}+00,2.74$ $\mathrm{E}+00$ and $1.71 \mathrm{E}+00 \mathrm{mg} / \mathrm{kg} /$ body (children) for samples from Osisioma, Irete, Nnewi, Ishiagu and Ngwo respectively.

The incremental life time cancer risk has a predicted permissible lifetime risks for carcinogens as $10^{-6}(1$ in $10,000,000)$ and $10^{-4}(1$ in 10,000$)$ range $[5,7,9,27,28]$. Values above this range indicate carcinogenic risk [9]. Considering the study population, the carcinogenic risk resulting from consumption of the variety of food crops for adults and children were 1.39 E-06, $1.54 \mathrm{E}-06$; 3.43 E-06, 3.79 E-06; 6.19 E-07, 6.87 E-07; 1.09 E-06,1.21 E06; and 9.71 E-07, 1.05 E-06 for Osisioma, Akwuuru, Irete, Ishiagu and Enugu industrial locations respectively (Table 3). From the above, CR values were within the predicted life time risks for carcinogens for adults and children in while that of Osisioma, Akwuuru and Ishiagu while CR values for Irete and Enugu were within safe limits. These values suggest that $\mathrm{CR}$ exposure through the intake of food cultivated the around industrialized areas may be attributed to the type and activities peculiar to each industry. For instance, petrochemical and mining activities at Osisioma, Akwuuru and Ishiagu study location may be responsible for the increase in $\mathrm{CR}$ value compared to Irete and Enugu where we have vegetable oil production and bottling company respectively. Hence, adults and children residing at industrial areas in South Eastern Nigeria may be at serious risk due to PAHs intake in foods overtime due to bioaccumulation of this toxic carcinogen.

\section{Conclusion}

There was a significant level of PAHs in food crops especially in vegetables and soils in the study areas. More than $50 \%$ of $\sum \mathrm{B}(\mathrm{a}) \mathrm{P}$ and $\sum \mathrm{PAHs}$ in food crops like Telferia occidentali, Elaies guinnensis, Cocos nucifera and Citrus sinensis across the study locations were higher than limits set by DPR $(0.01 \mathrm{mg} / \mathrm{kg})$ and $\mathrm{EU}(0.2 \mathrm{mg} / \mathrm{kg})$. The LMW-PAHS/HMW-PAHS were $<1$ for all the locations indicating that PAHs levels in foods is attributable to the industrial activities going on in the study locations. The human health risk assessment of PAHs via the ingestion of food crops ingestion of foods indicated that estimated daily intake of PAHs in samples were within the stipulated reference dose. The incremental life time cancer risk did not exceed the permissible limits $\left(10^{-6}\right.$ to $\left.10^{-4}\right)$ and thus may not be an issue of concern. However, the toxicity of PAHs acting together with other pollutants could constitute serious health hazard to humans. Therefore, prompt action is needed as the health of exposed population especially children may be seriously endangered overtime due to bioaccumulation of PAHs. Policy makers and other concerned stakeholders should also help in making recommendations and regulations in policy decisions and mitigating measures for environment and human health protections.

\section{Conflict of interest}

The authors declare no conflict of interest.

\section{References}

[1]. B.M. Omambia, G.M. Simiyu. Heavy metal $(\mathrm{Cu}$, $\mathrm{Zn}, \mathrm{Cd}$ and $\mathrm{Pb}$ ) contamination of Indigenous green vegetables in Eldoret town and their health implications to consumers, Research Conference paper, University of East Africa Baraton (2014). Retrieved from ueab.ac.ke on 07/02/2018

[2]. U.B. Onyedikachi, D.C. Belonwu, O.W. Mattew, Human health risk assessment of heavy metals in soils and commonly consumed food crops from quarry sites located at Isiagwu, Ebonyi State, Ovidius University Annals of Chemistry 29 (2018) 8-24.

[3]. M.D.S. Pereira, D. Heitmann, W. Reifenhäuser, O. M. Rodrigo,L.S Santos, J.P.M Torres,O. Malm, W. Körner, Persistent organic pollutants in atmospheric deposition and biomonitoring with Tillandsia usneoides (L.) in an industrialized area in Rio de Janeiro State, Southeast Brazil-Part II: PCB and PAHS, Chemosphere 67 (2007) 1736-1745

[4]. E.K. Ossai, C.M.A. Iwegbue, E.E Ajungungbe, G.O. Tesi, Polycyclic aromatic hydrocarbons and metal concentrations in imported canned maize, Turkish Journal of Agriculture - Food Science and Technology 3 (2015) 53-58.

[5]. E. Ohiozebau, B. Tendler, G. Codling, E. Kelly, J.P. Giesy, P.D. Jones, Potential health risks posed by polycyclic aromatic hydrocarbons in muscle tissues of fishes from the Athabasca and Slave Rivers, Canada, Environmental Geochemistry and Health, 39 (2017) 139-160. 
[6]. A. Paris, J. Ledauphin, P. Poinot, J.L. Gaillard, Polycyclic aromatic hydrocarbons in fruits and vegetables: Origin, analysis, and occurrence, Environmental Pollution 234 (2018) 96-106.

[7]. J. Zhang, C. Qu, S. Qi, J. Cao, C. Zhan, X. Xing X, Polycyclic aromatic hydrocarbons (PAHs) in atmospheric dustfall from the industrial corridor in Hubei Province, Central China, Environmental Geochemistry and Health 37 (2015) 891-903.

[8]. Y.X. Zhang, S. Tao, H.Z. Shen, J.M Ma, Inhalation exposure to ambient polycyclic aromatic hydrocarbons and lung cancer risk of Chinese population, Proceedings National Academy of Science U.S.A. 106 (2009) 21063-21067.

[9]. K.W. Nkpaa, M.O. Wegwu, E.B. Essien, Assessment of polycyclic aromatic hydrocarbons (PAHs) levels in two commercially important fish species from crude oil polluted waters of Ogoniland and their carcinogenic health risks, Journal of Environment and Earth Science 3 (2013) 128-137.

[10]. A.P.I, American Petroleum Institute, Inter laboratory study of three methods for analyzing petroleum hydrocarbons in soil, diesel range organics (DRO), gasoline range organics (GRO) and petroleum hydrocarbon (PHC), Number 4599 (1994).

[11]. E.O. Nwaichi, M.O. Wegwu, U.L. Nwosu, Distribution of selected carcinogenic hydrocarbon and heavy metals in an oil-polluted agriculture zone, Springer International Publishing Switzerland 186 (2014) 8697-8706.

[12]. E.O. Nwaichi, L.C. Chuku, E. Ighoavwogan, Polycyclic aromatic hydrocarbons and selected heavy metals in some oil polluted sites in Delta state Nigeria, Journal of Environmental Protection 7 (2016) 1389-1410.

[13]. I. Tongo, O. Ogbeide, L. Ezemonye, Human health risk assessment of polycyclic aromatic hydrocarbons (PAHs) in smoked fish species from markets in Southern Nigeria, Toxicology Report 4 (2017) 55-61.

[14]. A.T.S.D.R. Agency for Toxic Substances and Disease Registry (ATSDR).Minimal risk levels (MRLs) for hazardous substance, Washington, DC: ATSDR (1996).

[15]. J.C.T. Nisbet, P.K. La Goy, Toxic equivalence factors (TEFs) for polycyclic aromatic hydrocarbons (PAHs), Regulation Toxicology Pharmacology 16 (1992) 290-300.

[16]. P. Avila, S. Ferreira, C. Candeias, Health risk assessment through consumption of vegetables rich in heavy metals: the case study of the surrounding villages from Panasqueira mine, Central Portugal. Springer International Publishing Switzerland, (2016) 34. DOI 10.1007/s10653-016-9834-0

[17]. J.J. Slaski, D.J. Archambault, X. Li, Evaluation of polycyclic aromatic hydrocarbon (PAHs) accumulation in plants. The potential use of PAHs accumulation as a marker of exposure to air emissions from oil and gas flares, Report prepared for the Air Research Users Group, Alberta Environment, Edmonton, Alberta (2000).
[18]. S. Lu, L. Xiaoyong, Y. Xiulan, Z. Ganghui, M. Dong, Evaluation of heavy metal and polycyclic aromatic hydrocarbons accumulation in plants from typical industrial sites: potential candidate in phytoremediation for co-contamination, Environmental Science and Pollution Research 21 (2014) 12494-12504.

[19]. A. Luch, The carcinogenic effects of polycyclic aromatic hydrocarbons, London Imperial College Press (2005).

[20]. A. Soceanu, S. Dobrinas, V. Popescu, Polycyclic aromatic hydrocarbons in Romanian baby foods and fruits, Polycyclic Aromatic Compound 36 (2016) 364-375.

[21]. L.Z. Zhu, H. Lu, S.G. Chen, T. Amagai, Pollution level, phase distribution and source analysis of polycyclic aromatic hydrocarbons in residential air in Hangzhou, China, Journal of Hazard Materials 162 (2009) 1165-1170.

[22]. L.J. Wang, X. Xu, X.W. Lu, Composition, source and potential risk of polycyclic aromatic hydrocarbons (PAHs) in vegetable soil from the suburbs of Xianyang City, Northwest China: a case study, Environmental Earth Science 75 (2016) 113.

[23]. E. Inam, F. Ibanga, J. Essien, Bioaccumulation and cancer risk of polycyclic aromatic hydrocarbons in leafy vegetables grown in soils within automobile repair complex and environ in Uyo, Nigeria, Environmental Monitoring and Assessment 188 (2016) 1-9.

[24]. A. Abou-Arab, M. Abou-Donia, F. El-Dars, O. Ali, A. Hossam, Levels of polycyclic aromatic hydrocarbons (PAHs) in some Egyptian vegetables and fruits and their influences by some treatments, International Journal of Current Microbiology Applied Science 3 (2014) 277-293.

[25]. V. Akpambang, G. Purcaro, L. Lajide, I.A. Amoo, L.S. Conte, S. Moret, Polycyclic aromatic hydrocarbons in some Nigerian roasted plant foods, Front, Food Nutrition Research 1 (2015) 1-5.

[26]. X.T. Wang, L. Chen, X.K. Wang, B.L. Le, Y.F Sun, J. Zhou, M.H. Wu, Occurrence, sources and health risk assessment of polycyclic aromatic hydrocarbons in urban (Pudong) and suburban soils from Shanghai in China, Chemosphere 119 (2015) 1224-1232.

[27]. S. Chawda, A. Tarafdar, A. Sinha, B.K. Mishra, Profiling and health risk assessment of PAHs content in Tandoori and Tawa Bread from India, Polycyclic Aromatic Compounds 5 (2017) 1-12.

[28]. X. Wei, Y. Huang, M.H. Wong, M.H. Giesy, C.K.C. Wong, Assessment of risk to humans of bisphenol: A in marine and freshwater fish from Pearl River Delta, China, Chemosphere 85 (2011) 122-128.

Received: 16.03.2019

Received in revised form: 07.04.2019

Accepted: 10.04.2019 